CONSEIL D'ORIENTATION DES RETRAITES

Séance plénière du 20 octobre 2010 - 9 h 30

«Elaboration de cas types pour le pilotage du système de retraite »

Document $N^{\circ} 6$

Document de travail, n'engage pas le Conseil

Profils types des salariés du secteur privé : approche par une classification des carrières

Karine Briard - Economie et Prévision n 180-181, 2007/4-5 


\title{
Profils types des salariés du secteur privé : approche par une classification des carrières
}

\author{
Karine Briard $^{(*)}$
}

L'image traditionnelle du travailleur restant dans la même entreprise de la sortie des études à la retraite contraste avec celle renvoyée par le marché du travail depuis au moins deux décennies. Dans le secteur privé, les parcours professionnels sont scandés par des périodes de chômage et d'inactivité, la durée pour l'accès au premier emploi stable tend à se prolonger et le départ en retraite fait souvent suite à une période d'inactivité ...

Cette diversité de trajectoires professionnelles paraît si éloignée du modèle de la carrière continue qu'elle soulève la question de la pertinence de ce parangon. La « carrière continue » a-t-elle même jamais existé ? La multiplicité de parcours observée peut-elle être entièrement attribuée aux profonds changements socioéconomiques des dernières décennies avec lesquels le secteur privé est directement en prise comme la montée du chômage et le développement des formes flexibles d'emploi (contrat à durée déterminée, temps partiel, intérim ...) ? Si plusieurs types de carrière ont toujours coexisté, comment leur représentativité a t-elle évolué ? La carrière continue concernerait-elle moins de salariés qu'auparavant? Des profils de carrière jusqu'ici minoritaires ont-ils pris une importance croissante?

Pour répondre à ces questions, on se propose de mettre au jour des proximités dans les parcours professionnels suivis par les salariés du secteur privé. L'idée est de constituer des groupes de carrières au profil proche et de suivre l'évolution de ces groupes sur plusieurs générations. La démarche repose sur une analyse typologique des comptes individuels des assurés du régime d'assurance vieillesse de la Sécurité sociale, comptes gérés par la Caisse nationale d'assurance vieillesse (Cnav). Pour aborder de façon directe les progressions salariales et le déroulement des parcours professionnels, sans position explicative a priori, elle reprend les techniques exploratoires de l'analyse de données telles que l'analyse de classification et l'analyse discriminante.

L'analyse menée sur les comptes des ayants droit du Régime général nés entre 1935 et 1960 fait apparaître une typologie " transgénérationnelle 》 de sept groupes de parcours : la carrière salariale courte (1), celle effectuée en majorité hors du secteur privé (2), la carrière d'invalide (3), la carrière à salaires très faibles avec inactivité entre 25 et 35-40 ans, typique des mères de famille (4), la carrière précaire à salaires faibles, avec interruptions dues, entre autres, au chômage (5), la carrière continue avec des salaires inférieurs au plafond (6) et celle aux salaires proches du plafond (7). Un dualisme au sein du secteur privé apparaît, opposant des travailleurs aux conditions de travail précaires, certains vraisemblablement peu qualifiés, à des travailleurs moins exposés au chômage et mieux rémunérés.

(*) CNAV - Direction de l'actuariat statistique.

E-mail : karine.briard@cnav.fr

Pour leurs suggestions et remarques, je tiens à remercier les deux rapporteurs anonymes de la revue. Mes remerciements s'adressent également aux membres du jury de ma thèse dont cet article reprend la première partie : Florence Legros, directrice, Didier Blanchet et Marie-Claire Villeval, rapporteurs, Anne Lavigne et Jérôme Gautié. 
Les croisements de cette typologie avec des stratifications plus "classiques 》, par condition d'emploi, catégorie sociale et secteur d'activité, fournissent plusieurs éléments illustratifs de cette polarisation : en 2000, moins de $45 \%$ des salariés du groupe 4 occupaient un emploi à temps complet, $92 \%$ des salariés du groupe 7 ; les deux tiers des premiers étaient employés, les deux tiers des seconds appartenaient à la catégorie des «Professions intermédiaires » ou étaient cadres ; enfin, les premiers étaient sous représentés dans les secteurs offrant les meilleures rémunérations (Énergie, Activités financières et Industrie de biens intermédiaires et d'équipement), alors que les seconds y étaient majoritaires.

Au fil des générations, la progression des salaires au cours de la carrière s'est peu à peu infléchie. Mais si, entre 20 et 40 ans, les salariés du groupe 7 ont pu conserver une croissance de leurs salaires de plus de $6 \%$, les revenus annuels des salariés du groupe 4 qui croissaient de 2,3\% pour les générations 1935 à 1940, ne font plus que rester constants pour ceux nés dans la seconde moitié des années cinquante; en cause : l'allongement et la multiplication des périodes de chômage et le développement des emplois flexibles (Cdd, intérim, temps partiel).

Les transformations du marché du travail des dernières décennies déforment les profils de carrière et en modifient la représentativité. Le suivi de la typologie au fil des générations témoigne ainsi de la persistance d'un noyau dur de carrières continues, longues et à salaires élevés (de l'ordre de $20 \%$ des assurés), et d'une part croissante des carrières précaires, aux salaires faibles, marquées par des périodes de chômage à des âges de plus en plus jeunes; ces dernières prenant le pas sur les carrières les plus courtes pour lesquelles les interruptions d'activité relèvent plus probablement d'un choix que d'une contrainte.

À défaut de disposer de données détaillées pour des salariés nés dans l'Entre-deux-guerres, il reste difficile de dire si le modèle de la carrière continue de la sortie des études à la retraite a jamais été dominant. Les parcours suivis par les assurés nés entre 1935 et 1940 ne répondaient déjà plus à ce schéma, mais ils n'en déviaient qu'à l'approche de la retraite. La montée du chômage et le développement des formes flexibles d'emploi des dernières décennies ont mis en exergue une bipolarisation entre, d'une part, des carrières précaires au profil salarial peu croissant et, d'autre part, des carrières continues aux progressions salariales plus soutenues. Pour les générations de l'après baby boom, le modèle de la carrière longue et continue du salarié du secteur privé ne s'oppose désormais plus à celui d'une carrière brève où le retrait du marché du travail s'effectue précocement, mais à des parcours parfois plus longs et toujours plus discontinus. Dans cette évolution, le parcours des femmes s'améliore, alors que dans le même temps celui des hommes se dégrade. Les modèles d'activité des hommes et des femmes convergent, mais non vers le standard de la carrière continue, mais vers un parcours plus discontinu. 
L'image traditionnelle du travailleur restant dans la même entreprise de la sortie des études à la retraite contraste avec celle renvoyée par le marché du travail depuis au moins deux décennies, car les parcours professionnels des salariés du secteur privé sont scandés par des périodes de chômage et d'inactivité, la durée pour l'accès au premier emploi stable tend à se prolonger sur plusieurs années après les études, le départ en retraite fait souvent suite à une période d'inactivité déjà indemnisée (préretraite, chômage) ...

Cette diversité de trajectoires professionnelles paraît si éloignée du modèle de la carrière continue qu'elle soulève la question de la pertinence de ce parangon. La « carrière continue » a t-elle même jamais existé ? La multiplicité de parcours observée peut-elle être entièrement attribuée aux profonds changements socioéconomiques des dernières décennies avec lesquels le secteur privé est directement en prise, la montée du chômage et le développement des formes flexibles d'emploi (contrat à durée déterminée, temps partiel, intérim, ...) ? Si plusieurs types de carrière ont toujours coexisté, comment leur représentativité a t-elle évolué? La carrière continue concernerait-elle moins de salariés qu' auparavant? Des profils de carrière jusqu'ici minoritaires ont-ils pris une importance croissante?

Les travaux présentés dans cet article visent à avancer sur ces questions en mettant au jour des proximités dans les parcours professionnels suivis par les salariés du secteur privé, constituer des groupes de carrières au profil proche et suivre l'évolution de ces groupes sur plusieurs générations.

La démarche repose sur une analyse typologique des comptes individuels des assurés du régime d'assurance vieillesse de la Sécurité sociale auquel ces salariés sont affilés de façon obligatoire. L'analyse directe des progressions salariales et des séquences d'états de participation au marché du travail (salariat dans le privé, chômage, activités hors du secteur privé, invalidité, etc.) permet alors de se départir d'une position explicative a priori.

La première partie de cet article rappelle l'intérêt d'une telle approche et précise sa mise en œuvre sur le fichier des cotisants de la Caisse nationale d'assurance vieillesse (Cnav), gestionnaire du régime général d'assurance vieillesse. La deuxième partie s'attache à présenter la typologie de carrières mise au jour par l'analyse de classification. Elle s'intéresse en premier lieu aux salariés nés entre 1935 et 1940 dont le parcours peut être observé jusqu'à son terme, puis regarde dans quelle mesure chacun des profils de carrière identifié persiste sur les générations suivantes. Enfin, la troisième partie revient sur les caractéristiques des différents types de carrière en portant une attention particulière aux carrières salariales (niveau et évolution des salaires) et à la vulnérabilité au chômage (fréquence et durée).
Les croisements de la typologie avec des stratifications plus « classiques », par condition d'emploi, catégorie sociale et secteur d'activité, complètent cette analyse descriptive et viennent alimenter les pistes permettant d'expliquer comment, de génération en génération, les parcours professionnels ont pu progressivement s'éloigner du modèle de la carrière continue.

\section{Méthodologie}

\section{De l'intérêt d'une analyse longitudinale et typologique}

La mise en évidence empirique de parcours professionnels types qui seraient les représentants de segments du marché du travail régis par des mécanismes de rémunération, d'accession et de sécurité de l'emploi différents, semble facilitée par les différents angles sous lesquels elle peut être abordée, car les signes d'une segmentation sont multiples : attachement à l'emploi, fréquence et durée du chômage, politiques de gestion du personnel privilégiant la flexibilité ou, au contraire, incitant à rester dans l'entreprise par un système de primes à l'ancienneté ou de promotions internes, technologie de production requérant une main d'œuvre qualifiée, etc.

Toutefois, les caractéristiques individuelles à elles seules ne sont pas suffisantes pour définir des segments distincts et porteurs de sens. Bayet et Colin (1998) rappellent que « selon l'entreprise d'emploi, les perspectives d'évolution ne sont pas du tout les mêmes. Ceci tient au fait que toutes les entreprises ne sont pas soumises aux mêmes conditions de marché, aux mêmes conditions techniques de production, ni aux mêmes forces institutionnelles ou exigences des actionnaires ». La segmentation par emplois pourrait ainsi fournir un cadre d'analyse plus adéquat en permettant de contrôler les mécanismes de rémunération. Lemistre et Plassard (1998) remarquent d'ailleurs que la relative homogénéité des carrières des travailleurs occupant le même type d'emploi semble confirmée pour la France et, citant Le Gall, «les salariés sont [en effet] situés et évalués par l'emploi qu'ils occupent dans l'entreprise, autant et parfois plus que par leurs caractéristiques personnelles ${ }^{(1)}$ ». Sauf à s'intéresser à un champ d'étude très spécifique - une entreprise ou une branche, par exemple -, la carence de données en la matière compromet néanmoins ce type d'analyse.

Face à la diversité et à l'imbrication des variables de stratification requises pour mettre en évidence des profils de carrières différenciés, résultats de différents mécanismes de rémunération et de rapport à l'emploi, le suivi des parcours individuels apparaît comme la solution la plus fiable. Lorsque l'approche 
exploratoire est rendue possible par la disponibilité de données longitudinales, elle permet en outre de s'affranchir d'une position explicative a priori et d'appréhender les trajectoires dans leur globalité et non pas seulement en certains points du temps comme le font les modèles stochastiques traditionnels (séries temporelles, chaînes de Markov, analyse biographique) en se focalisant sur les transitions entre évènements isolés.

Le parcours professionnel peut être vu comme un enchaînement de séquences d'états. La variété des états, la diversité des combinaisons d'états successifs possibles et la durée de chacun d'eux, font $\mathrm{du}$ parcours un objet complexe à décrire. Une solution consiste néanmoins à postuler que les parcours individuels sont identifiables à des phrases. Les techniques d' analyse typologique reposent alors sur les méthodes développées en analyse de données textuelles (Benzécri, 1981 ; Lebart et Salem, 1988), lesquelles consistent à rechercher des séquences communes de « lettres », d'états, dans un ensemble de parcours observés empiriquement puis codés (cf. annexe 1).

L'analyse typologique des parcours (ou analyse de séquences) reste mal connue, probablement pour deux raisons, néanmoins contestables : la défiance à l'égard des techniques d'analyse de données, parfois mal employées, et la dominance de l'économétrie, explicative, dont elle est pourtant complémentaire et devrait être un préalable.

Les analyses de séquences sont des techniques descriptives qui permettent d'étudier les similitudes entre différents parcours en mettant en évidence des régularités dans la structure, le déroulement, des parcours observés. En revanche, elles ne produisent aucun résultat sur les corrélations entre l'apparition d'un événement $\mathrm{A}$ et celle d'un événement $\mathrm{B}$ survenant ultérieurement. Pour rechercher les facteurs expliquant l'occurrence d'une forme particulière, la modélisation économétrique se présente alors comme la suite naturelle à l'analyse typologique.

Fénelon et alii (1997) distinguent d'ailleurs les approches typologique et économétrique par leur objet et la place qu'elles occupent dans l'analyse : [...] la préoccupation principale, dans la démarche typologique, est de chercher des régularités dans la diversité des trajectoires, pour en proposer une synthèse qui aura statut de variable de résultat. C'est donc une étape intermédiaire entre la mise en forme des données et l'estimation d'un modèle : mise en forme de la trajectoire qui aboutit [...] à proposer une variable de résultat synthétique élaborée. Ce qu'on modélise, c'est la trajectoire, et non ses liens avec d'autres variables individuelles. Alors que dans la démarche économétrique, la variable de résultat est identifiée d'emblée, le critère de l'évaluation est clairement posé, et la modélisation porte sur ses liens, plus ou moins sophistiqués, avec d'autres caractéristiques de l'individu.

L'intérêt de faire précéder l'analyse économétrique d'une analyse typologique serait donc double: ne pas imposer de schéma théorique a priori, mais, au contraire, guider la spécification du modèle explicatif ; " explorer l'explicatif », " tout voir et retenir l'essentiel » (Bry et Antoine, 2004).

Toutefois, si les combinaisons entre analyse typologique et analyse économétrique sont potentiellement multiples, les études reliant les deux approches restent rares. Les méthodes d'analyse de parcours restent finalement scindées en deux grandes familles, chacune répondant à un objectif précis (Fénelon et alii, op. cit.) : d'une part, les méthodes qui relèvent d'une approche descriptive et typologique, avec pour but de synthétiser l'hétérogénéité des situations individuelles, prises dans leur globalité, puis d'en identifier quelques formes représentatives en posant un minimum d'hypothèses ; d'autre part, les méthodes qui relèvent de l'économétrie et qui consistent à modéliser les parcours en estimant les effets des variables retenues comme explicatives et la loi de distribution attribuée à la réalisation de tel(s) ou tel(s) événement(s).

L'approche typologique se justifie néanmoins à elle seule lorsque l'objectif n'est pas explicatif, mais exploratoire, descriptif ; lorsqu'il s'agit, par exemple, de déterminer un petit nombre de parcours professionnels censés représenter ceux suivis par plusieurs millions d'individus, comme c'est l'objet de nos travaux.

\section{Une analyse de données textuelles sur les comptes individuels des assurés du Régime général}

Les reports effectués, année après année, sur les comptes individuels des assurés du Régime général se prêtent tout particulièrement à une analyse longitudinale des parcours professionnels des salariés du secteur privé. Un important travail de mise en forme des données et de développement de procédures statistiques garantissant la robustesse des analyses doit cependant être réalisé.

\section{L'unité d'analyse : le compte individuel de l'assuré du Régime général}

La Cnav archive les carrières de plus de soixante millions d'assurés depuis 1947 et l'échantillon au $1 / 20$ du fichier national des cotisants extrait en mai 2002, représente une base de données de deux millions de comptes individuels pour des assurés nés entre 1935 et 1985 . Il y est fait mention des éléments servant au calcul de la retraite personnelle, tels que : - les séries de salaires annuels soumis à cotisations (salaires au plus égaux au salaire plafond de la Sécurité sociale, 32184 euros par an au $1^{\mathrm{er}}$ janvier 2007), lesquelles sont issues des déclarations 
annuelles de données sociales des employeurs (DADS) ;

- les périodes non cotisées (dites «périodes assimilées», PA), validées au titre du service national, au titre du chômage, de la maladie et de l'invalidité, ces dernières étant signalées par les Assedic et les caisses primaires d'assurance maladie ;

- les périodes validées par les régimes d'assurance vieillesse ayant passé des conventions de partage de données avec la Cnav (régimes agricoles MSA, régime des collectivités locales CNRACL, des artisans Cancava, des commerçants Organic ${ }^{(2)}$, etc.) et couvrant près de $90 \%$ des cotisants à des régimes de base français.

En raison de la fiabilité et de la richesse de ses informations, ce fichier de données longitudinales est une source de référence pour les statisticiens et actuaires de la Cnav. Il sert notamment à constituer la base de simulation du modèle de projection financière du régime et présente des avantages évidents pour l'étude socioéconomique des parcours professionnels en renseignant sur la nature des interruptions de carrière. Cependant, dédié au calcul des droits à retraite et non à l'étude économique des parcours professionnels, il présente aussi plusieurs inconvénients qui tiennent aux données disponibles et au champ couvert.

\section{Des données socioéconomiques rares}

L'analyse économique de la formation des salaires et de la participation au marché du travail repose classiquement sur des variables retraçant aussi bien des caractéristiques individuelles, telles que le sexe, la nationalité ou le niveau de capital humain (diplôme, expérience, ancienneté dans l'emploi ou dans l'entreprise, ...), que des caractéristiques collectives, telles que la branche d'activité, la taille de l'entreprise (effectifs, chiffres d'affaires, ...), la localisation géographique, etc ${ }^{(3)}$.

Pour la plupart, ces variables sont absentes du fichier-échantillon de cotisants de la Cnav. Les informations sur le secteur d'activité et la catégorie socioprofessionnelle (PCS) ne sont, quant à elles, enregistrées que depuis 1999. Par ailleurs, renseignée à partir des DADS, la PCS n'est disponible que pour les entreprises d'au moins 20 salariés et la mention reste facultative pour les petites entreprises, lesquelles représentent plus du quart du salariat privé(4), la taille de l'entreprise n'est cependant pas précisée.

Des informations partielles sur les salaires et la durée d'activité

Les salaires sont reportés au compte individuel dans la limite du plafond soumis à cotisations. Cette troncature concerne un nombre non négligeable de comptes, car jusqu'à près de la moitié des assurés nés en 1940 - certes, la génération la plus concernée - a perçu au moins un salaire supérieur ou égal au plafond au cours de la carrière (Debrand et Privat, 2002 ; Koubi, 2002).

La durée d'activité, annuelle ou journalière, n'est pas mentionnée de façon explicite. La condition d'emploi (temps complet, temps partiel, intermittence ou travail à domicile) n'est renseignée que depuis 1999, d'où l'impossibilité de distinguer les activités à temps partiel de celles exercées une partie de l'année. La durée d'activité annuelle ne peut donc être que grossièrement estimée à partir de la durée d'assurance validée, exprimée en trimestres ${ }^{(5)}$, celle-ci pouvant être acquise de trois façons :

- par cotisations, à raison d'un trimestre d'assurance pour autant de fois que le salaire brut représente le salaire de référence, l'équivalent de 200 heures rémunérées au Smic ;

- par «assimilation» à des périodes de cotisation, au titre de certaines situations d'interruption de travail : un trimestre accordé en cas d'accouchement, en cas de maladie ou d'accident du travail pour autant de fois 60 indemnités journalières, à toute échéance du paiement trimestriel de la pension d'invalidité, pour 50 jours de chômage ou encore pour 90 jours de service militaire (service national, mobilisation ou engagement volontaire en temps de guerre $)^{(6)}$;

- au regard de la situation personnelle et familiale de l'individu : des trimestres de majoration accordés aux femmes assurées pour chacun de leur enfant, d'autres aux assurés ayant pris un congé parental ou liquidant leur droits à retraite au-delà de l'âge de 65 ans.

Des informations lacunaires sur les périodes d'activité effectuées hors du secteur privé

Les périodes de service militaire et d'activité professionnelle effectuée hors du secteur privé (essentiellement dans la fonction publique et à l'étranger) sont reportées tardivement au compte individuel de l'assuré, le plus souvent lors du calcul de ses droits à retraite. En conséquence, les comptes de cotisants, non encore retraités, peuvent être lacunaires et ce risque est d'autant plus élevé que les assurés sont jeunes.

L'Échantillon interrégimes de retraités (EIR) ${ }^{(7)}$ a permis d'estimer qu'en 2001, $5 \%$ des retraités du Régime général étaient également prestataires de la fonction publique. En 2003, près de $8 \%$ des prestataires d'un droit direct au Régime général résidaient à l'étranger (Pascal, 2005), la possibilité que certains aient pu y occuper un emploi n'étant pas exclue. Par conséquent, jusqu'à $13 \%$ des comptes individuels pourraient ne pas faire mention de ces périodes d'activité. 


\section{Mise en œuvre de l'analyse sur les comptes des assurés du Régime général}

\section{La population étudiée}

Les travaux de classification présentés dans la suite de cet article portent sur les comptes individuels, arrêtés au 31 décembre 2000, des ayants droit survivants au 31 décembre 2001, qui ont reporté entre 15 et 65 ans au moins un salaire supposé correspondre à une activité régulière dans le secteur privé, sans interruption ou prise d'activité en cours d'année ${ }^{(8)}$. Une année donnée, les individus étudiés peuvent être actifs dans le secteur privé, au chômage, inactifs pour cause de maladie ou d'invalidité, ou encore être sortis du champ du Régime général. L'analyse se restreint ainsi au secteur privé, mais selon une acception large : sont observés tous les individus ayant transité pour une période plus ou moins longue par le secteur privé entre leurs $15^{\mathrm{e}}$ et $64^{\mathrm{e}}$ anniversaires, seule la période $15-59$ ans étant toutefois étudiée ${ }^{(9)}$.

L'analyse des parcours comprend deux grandes phases : leur représentation, ou « codage », et leur classification (cf. compléments en annexe 1).

\section{La représentation des parcours : le "codage"}

L'étape principale de l'analyse réside dans la constitution du « vocabulaire » servant à décrire les parcours individuels. Il s'agit de définir une nomenclature d'états - « un alphabet » - et la façon de regrouper ces états en segments porteurs de sens « des mots »-de façon à réduire la dimension des données tout en conservant la richesse de l'information (nature et succession des étapes, durée passée dans chacune d'elles, date à laquelle elles surviennent). L'étape est essentielle puisqu'elle détermine les éléments à partir desquels seront constitués et caractérisés les groupes censés partitionner de façon pertinente la «population » de parcours.

L'approche retenue est étroitement liée à l'objectif poursuivi ; selon que l'on souhaite mettre l'accent sur l'enchaînement des épisodes ou sur la date de la survenue d'un événement particulier, les choix méthodologiques seront différents. Les diverses méthodes d'analyse typologique des parcours se distinguent d'ailleurs principalement par les différences dans le codage et les choix de synthèse de l'information (Checcaglini et Lemaire, 2001).

La procédure de codage adoptée pour analyser les comptes des assurés du Régime général est décrite dans l'annexe 2 .

\section{La procédure de classification}

La seconde étape de l'analyse typologique consiste en la classification des carrières « codées ».

Sont ici enchaînées une analyse factorielle des correspondances multiples (AFCM), une classification mixte (algorithme non-hiérarchique des k-moyennes avec sélection des formes stables suivi d'une classification ascendante hiérarchique) puis une analyse discriminante ; l'AFCM sur les neuf variables qualitatives qui décrivent les sous-périodes quinquennales constituant les carrières, la classification sur les vingt facteurs principaux issus de l'AFCM, l'analyse discriminante sur les groupes issus de la classification.

Le nombre de groupes est déterminé par la confrontation de différents critères statistiques tels que le $R^{2}$ semi-partiel ou le pseudo $T^{2}$ qui renseignent sur la perte d'homogénéité induite par la réduction du nombre de groupes.

L'arbitraire de l'analyse est ainsi limité et la robustesse de la partition est assurée à trois niveaux de la procédure :

- les variables :

la classification est effectuée sur les axes principaux d'une analyse factorielle : les éventuelles fluctuations aléatoires sont ainsi renvoyées aux facteurs contribuant le moins à la variance de l'ensemble observé et l'orthogonalité des variables sur lesquelles est lancée la classification limite l'apparition de biais ;

- les méthodes :

en particulier, l'enchaînement des algorithmes de classification non-hiérarchique et hiérarchique permet de pallier les défauts de chacun d'eux ${ }^{(10)}$ et de converger vers une partition solide constituée de classes emboîtées ;

- les individus :

l'algorithme non-hiérarchique des k-moyennes est lancé trois fois avec des centres initiaux différents. La classification hiérarchique est ensuite réalisée sur les individus appartenant aux groupes formés par le croisement des trois partitions et regroupant au moins $2 \%$ des individus. Les individus alors exclus (au plus $30 \%$ de la population) sont affectés à l'un des groupes lors de l'analyse discriminante, ultime étape de la procédure de classification.

Afin d'apprécier dans quelle mesure un profil de carrière ou une typologie persiste d'une génération à l'autre, deux types d'analyses sont menés.

Dans un premier temps, cinq analyses de classification sont réalisées sur les comptes, observés jusqu'aux âges de 60, 55, 50, 45 et 40 ans, pour identifier les profils de carrière propres aux salariés des générations 1935 à 1940, 1941-1945, 1946 à 1950, 1951-1955 et 1956 à 1960 respectivement $^{(11)}$. Réalisées indépendamment les 
unes des autres, ces classifications ne sont pas directement comparables et ne permettent donc pas d'apprécier l'évolution de la part de chacun des groupes de parcours dans la population - leur représentativité - d'un ensemble de générations à l'autre pour se prononcer sur l'éventuelle persistance des profils de carrière.

Dans un second temps, une analyse discriminante est donc menée pour chacune des typologies identifiées en retenant une durée d'observation identique pour toutes les générations ${ }^{(12)}$, une partie de la carrière des salariés des générations les plus anciennes étant dès lors négligée. De cette façon, cinq analyses discriminantes sont réalisées, permettant de comparer de deux à cinq ensembles de générations. Dans le cas le plus critique, celui de la typologie des carrières des salariés des générations 1935 à 1940 observées jusqu'à 39 ans et non plus 59 ans, un tiers des individus est mal classé (cf. tableau 1). Ce taux reste raisonnable en comparaison de la probabilité de se tromper de groupe en procédant par hasard $(87,5 \%)$ et il tend plutôt à confirmer la prégnance des premières années de carrière sur la suite de la carrière.

L'analyse menée sur les cinq ensembles de générations relie les groupes de la typologie établie pour les salariés nés entre 1935 et 1940, observés jusqu'à l'âge de 60 ans, aux parcours professionnels des salariés nés entre 1935 et 1960, observés jusqu'à l'âge de 40 ans, soit les 25 premières années de carrière. Ce sont les groupes de carrières redéfinis par cette analyse qui seront présentés dans la dernière partie de cet article.

\section{Une typologie transgénérationnelle de carrières}

Un consensus émerge depuis les années quatre-vingt, selon lequel la montée du chômage, de l'activité féminine et la modification des pratiques de gestion du personnel auraient fait dévier les salariés du secteur privé de la route linéaire et balisée que leurs aînés suivaient. En particulier, les repères d'âges associés à l'accès à l'emploi stable et à la sortie du marché du travail auraient laissé place à des phases à part entière du cycle de vie : 1' « insertion » (Vincens, 1997) et la « décennie de la retraite» selon l'expression de Gaullier (1994). La période intermédiaire consacrée à l'emploi se serait raccourcie et le profil de rémunération continu et croissant se serait " éclaté » en une diversité de trajectoires $^{(13)}$.

Déterminer la génération à partir de laquelle le modèle de la carrière continue serait devenu minoritaire nécessiterait d'étudier les parcours professionnels de salariés ayant achevé leur vie active avant que n'apparaissent les profondes mutations affectant le marché du travail, au plus tard, dans la première moitié des années quatre-vingt.

La génération 1935, atteignant l'âge de 60 ans en 1995 , est la première génération que nous puissions suivre sur nos données. Si le point de référence s'en trouve reculé, la problématique de la recherche de carrières types pour les salariés du secteur privé et d'une éventuelle remise en cause du modèle dominant reste néanmoins posée : le parcours standardisé était-il vraiment pertinent pour les salariés aujourd'hui en âge de partir en retraite ? Peut-on identifier - et caractériser - d'autres parcours typiques pour ces assurés du Régime général ? Au fil des générations, ces parcours se déforment-ils, font-ils place à des types nouveaux ? Dans quelle mesure persistent-ils ?

Tableau 1 : taux de mauvais classement de l'affectation de carrières à des typologies définies sur différentes périodes d'observation

\begin{tabular}{|c|c|c|c|c|c|c|}
\hline \multirow{3}{*}{ Période } & \multirow{3}{*}{ Durée } & \multicolumn{5}{|c|}{ Typologie des carrières } \\
\hline & & à 40 ans & à 45 ans & à 50 ans & à 55 ans & à 60 ans \\
\hline & & \multicolumn{5}{|c|}{ Générations de référence } \\
\hline & d'observation & $1956-1960$ & 1951-1955 & $1946-1950$ & 1941-1945 & $1935-1940$ \\
\hline $15-39$ ans & 25 ans & \multirow[t]{5}{*}{$20 \%$} & $20 \%$ & $21 \%$ & $29 \%$ & $33 \%$ \\
\hline $15-44$ ans & 30 ans & & $15 \%$ & $14 \%$ & $20 \%$ & $22 \%$ \\
\hline $15-49$ ans & 35 ans & & & $10 \%$ & $14 \%$ & $15 \%$ \\
\hline $15-54$ ans & 40 ans & & & & $12 \%$ & $11 \%$ \\
\hline $15-59$ ans & 45 ans & & & & & $10 \%$ \\
\hline
\end{tabular}

Lecture : $33 \%$ des salariés nés entre 1935 et 1940, sélectionnés par l'algorithme de classification, ne sont pas affectés à leur groupe (tel qu'il a été déterminé à partir de leur carrière observée jusqu'à l'âge de 60 ans) si on se restreint à regarder les seules vingt-cinq premières années de leur parcours. 


\section{La référence : les carrières de 15 à 60 ans des salariés nés entre 1935 et 1940}

L'analyse de classification des parcours professionnels des travailleurs passés par le secteur privé et nés entre 1935 et 1940 distingue sept groupes $^{(14)}$ :

Groupe 1 : carrière salariale courte

Groupe 2 : carrière effectuée en majorité hors du secteur privé

Groupe 3 : carrière avec sortie du marché du travail par invalidité

Groupe 4 : carrière à salaires très faibles avec inactivité entre 25 et 35-40 ans

Groupe 5 : carrière précaire avec interruptions, à salaires faibles et chômage

Groupe 6 : carrière continue avec salaires inférieurs au plafond

Groupe 7 : carrière longue et continue avec salaires proches voire au plafond (et au-dessus).

La représentativité de ces groupes est très inégale. Le groupe 1 rassemble près du tiers de l'effectif total, le groupe 5, près du cinquième. À l'opposé, le groupe 3 représente moins de $1 \%$ de la population.

Parmi ces sept groupes, trois grandes catégories se distinguent en terme de participation au secteur privé : les carrières courtes, les carrières discontinues et les carrières longues et continues (figure 1).

\section{Les carrières courtes}

Les carrières courtes dans le secteur privé se concentrent dans les groupes 1,2 et 3 . En moyenne, la carrière salariale relevant du Régime général dure moins de dix ans (figure 2) et se situe en début de parcours professionnel comme en témoignent les taux de report de salaire dans le secteur privé (figure 1).

Le groupe 1 concerne $30 \%$ des salariés nés entre 1935 et 1940. Il s'agit pour l'essentiel de personnes dont la carrière s'est limitée au secteur privé ou qui ont poursuivi leur carrière dans la fonction publique ou à l'étranger. En effet, près de $40 \%$ des individus du groupe ont exercé une activité professionnelle hors du secteur privé qui n'a pas été mentionnée à leur compte avant leur départ en retraite, d'une durée de 25 à 35 ans pour la moitié d'entre eux. En 2002, le quart des retraités du groupe réside à l'étranger. Par différence, $15 \%$ des effectifs du groupe relèveraient ainsi de la fonction publique, soit $4 \%$ des assurés nés entre 1935 et 1940, chiffre proche des $5 \%$ estimés sur l'Échantillon interrégimes de retraités.
Les individus du groupe 2 ont une carrière longue, mais qui s'est déroulée en grande partie hors du secteur privé (29 années en moyenne). L'âge médian de sortie du champ du Régime général y est de 27 ans.

Enfin, la plupart des individus du groupe 3 sortent du marché du travail pour cause de maladie ou d'invalidité : en moyenne, entre 15 et 60 ans, les individus présentent 6 années avec au moins 60 jours de maladie (durée nécessaire pour valider un trimestre d'assurance au Régime général) et plus de 20 années en invalidité (figure 2).

\section{Les carrières discontinues}

Les carrières des groupes 4 et 5 sont moyennement longues. Elles comptent respectivement 21 et 23 années de salariat dans le secteur privé (figure 2) et présentent un pic d'activité entre 35 et 40 ans (figure 1).

Le groupe 4 se caractérise par un fléchissement des taux de salariat entre 20 et 35 ans. Le groupe est composé à $80 \%$ de femmes et le profil de carrière est caractéristique des mères de famille qui suspendent leur activité pour élever leurs enfants et qui réintègrent le marché du travail lorsque ceux-ci sont plus autonomes.

Les salariés du groupe 5 présentent également des taux de présence dans le secteur privé relativement faibles, hormis autour de l'âge de 40 ans où ils peuvent atteindre $80 \%$. Les femmes ne représentent plus que la moitié des effectifs. Des périodes d'inactivité - ou tout au moins des périodes d'activité non régulière - ponctuent le parcours professionnel et les individus connaissent en moyenne près de 4 années avec du chômage (figure 2).

\section{Les carrières longues et continues}

Les individus des groupes 6 et 7 ont des carrières longues et relativement continues; le taux de salariat atteint $70 \%$ dès 25 ans et dépasse ce niveau jusqu'à 50 ans. Les deux groupes se différencient principalement par le montant de leurs salaires, lequel atteint un maximum autour de l'âge de 40 ans : pour les individus du groupe 6 , il représente alors en moyenne $80 \%$ du plafond, alors que la quasi-totalité des individus du groupe 7 atteint voire dépasse le plafond (figure 4). Le service national interrompt le parcours professionnel en début de vie active, le chômage à l'approche de l'âge de la retraite : près de la moitié des travailleurs des groupes 6 et 7 sont au chômage à l'âge de 58 ans et, dans le groupe 6 , plus de $20 \%$ dès 55 ans; en moyenne, dans ce groupe, le chômage porte sur 3,2 années de la carrière et sur 2,6 dans le groupe 7 . 
Figure 1 : carrières de 15 à 60 ans des salariés nés entre 1935 et 1940

répartition des salariés de chaque groupe selon leur âge et ...

leur activité

leur niveau de salaire

Groupe $1:$ : carrière courte » $(30 \%$ des effectifs $-61 \%$ de femmes $)$
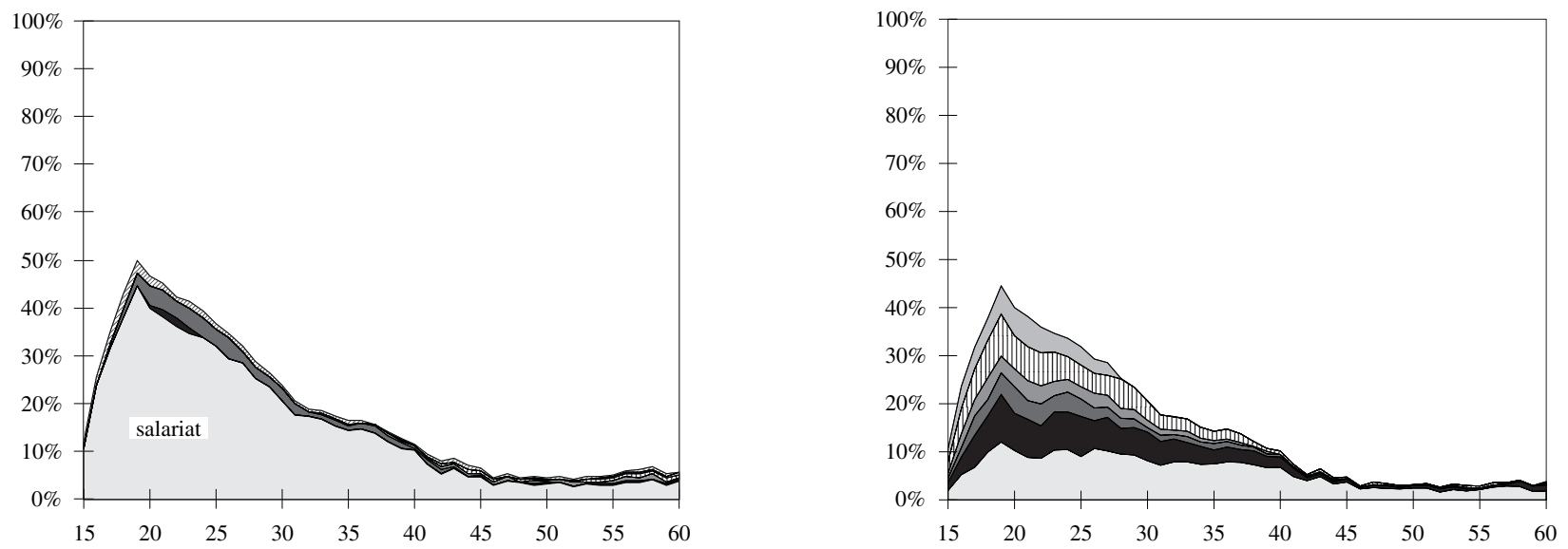

Groupe 2 : « hors secteur privé » $(14 \%-41 \%)$
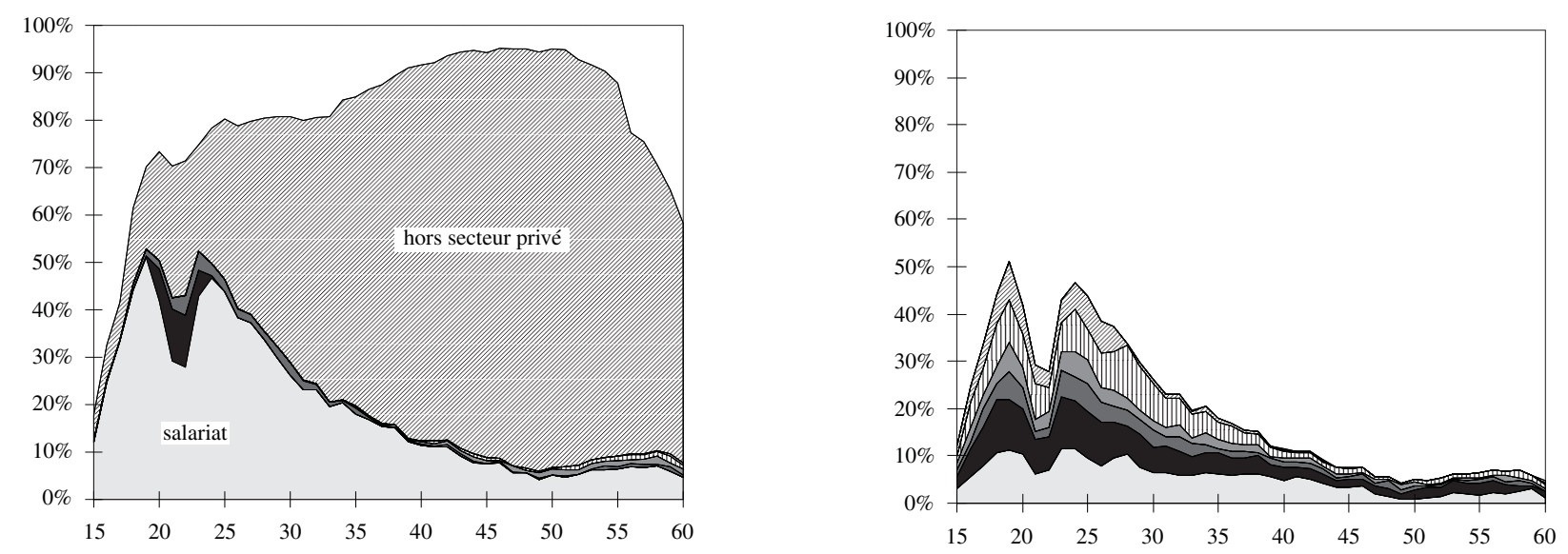

Groupe 3 : « invalide » $(1 \%-69 \%)$
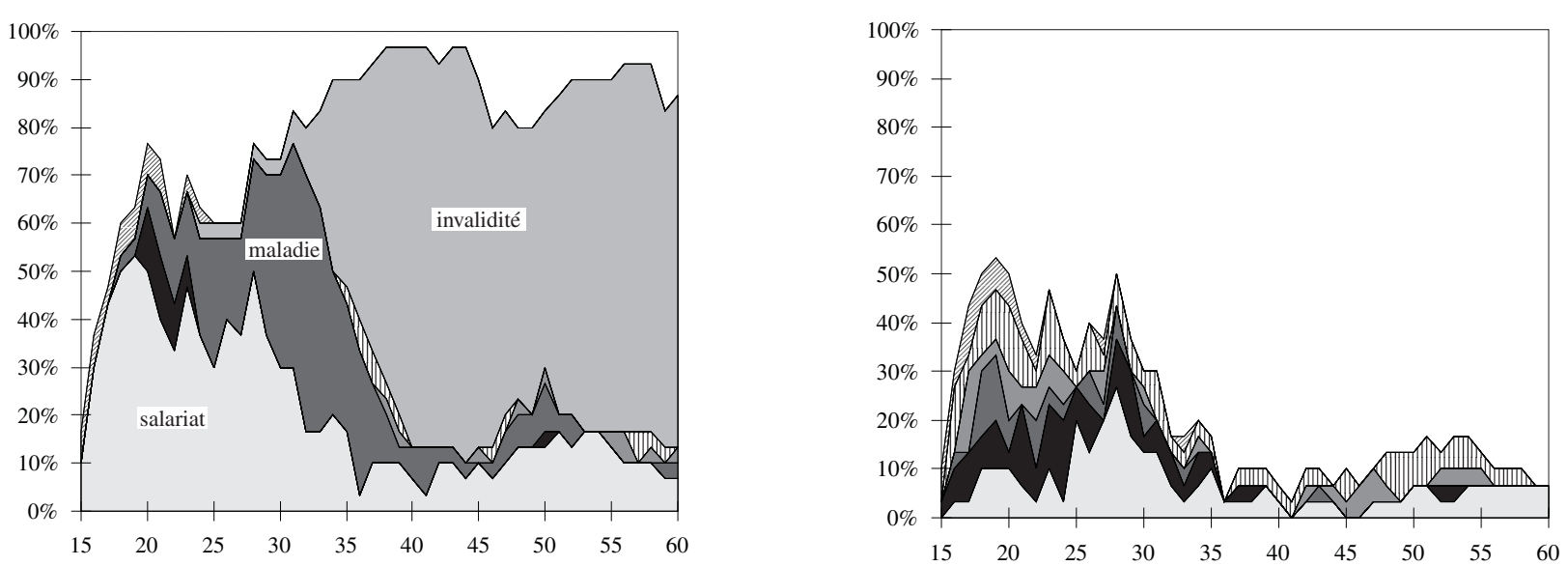

Note de lecture : à 25 ans, $44 \%$ des individus du groupe 2 sont salariés dans le secteur privé, $2 \%$ ont connu au moins 60 jours de maladie dans l'année (durée validant un trimestre), $34 \%$ travaillent hors du secteur privé et $20 \%$ sont inactifs ; pour $9 \%$ des individus du groupe, le salaire annuel est au plus égal au $2^{e}$ décile des salaires reportés par les salariés de leur génération (code Sb). 
Groupe 4 : « interrompue durablement à salaires bas» $(8 \%-90 \%)$
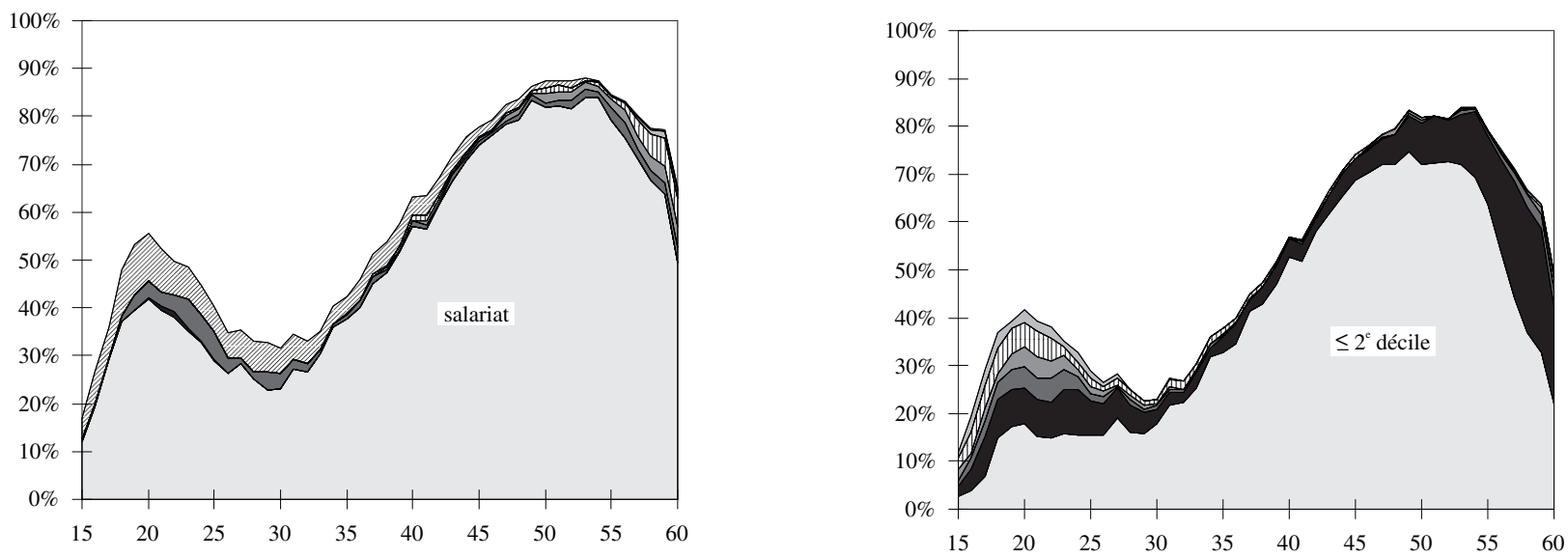

Groupe 5 : «précaire» $(18 \%-55 \%)$
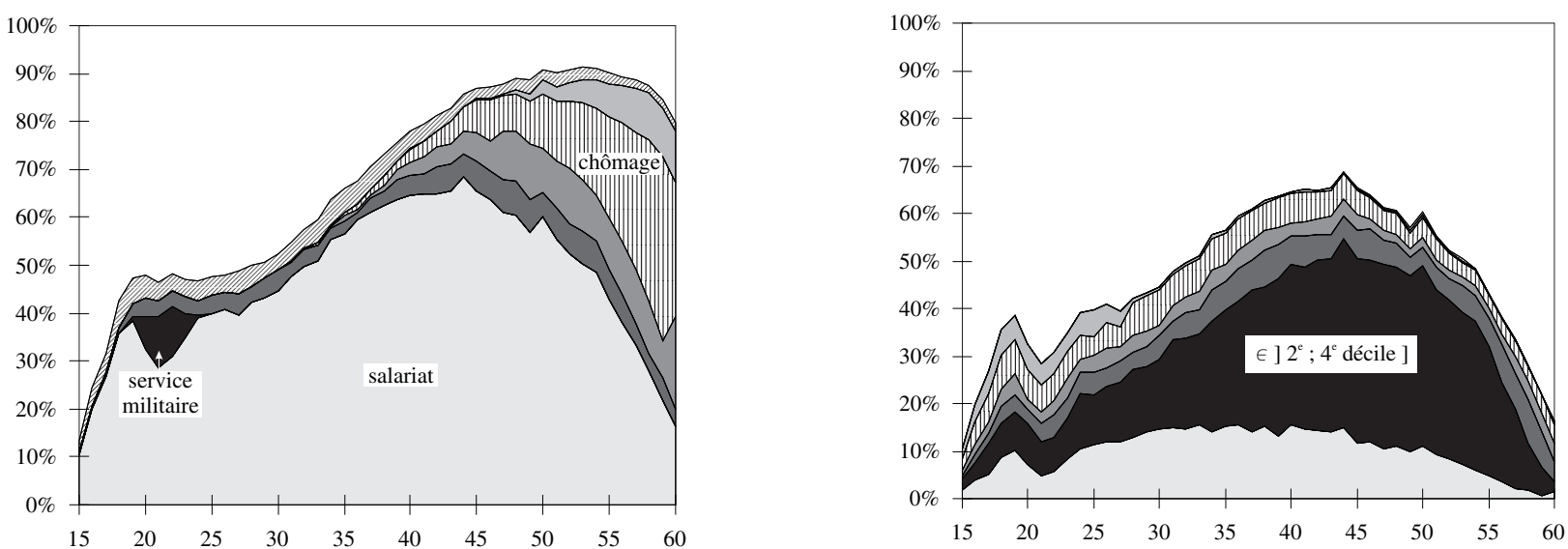

Groupe 6 : « continue à salaires sous plafond » $(12 \%-32 \%)$
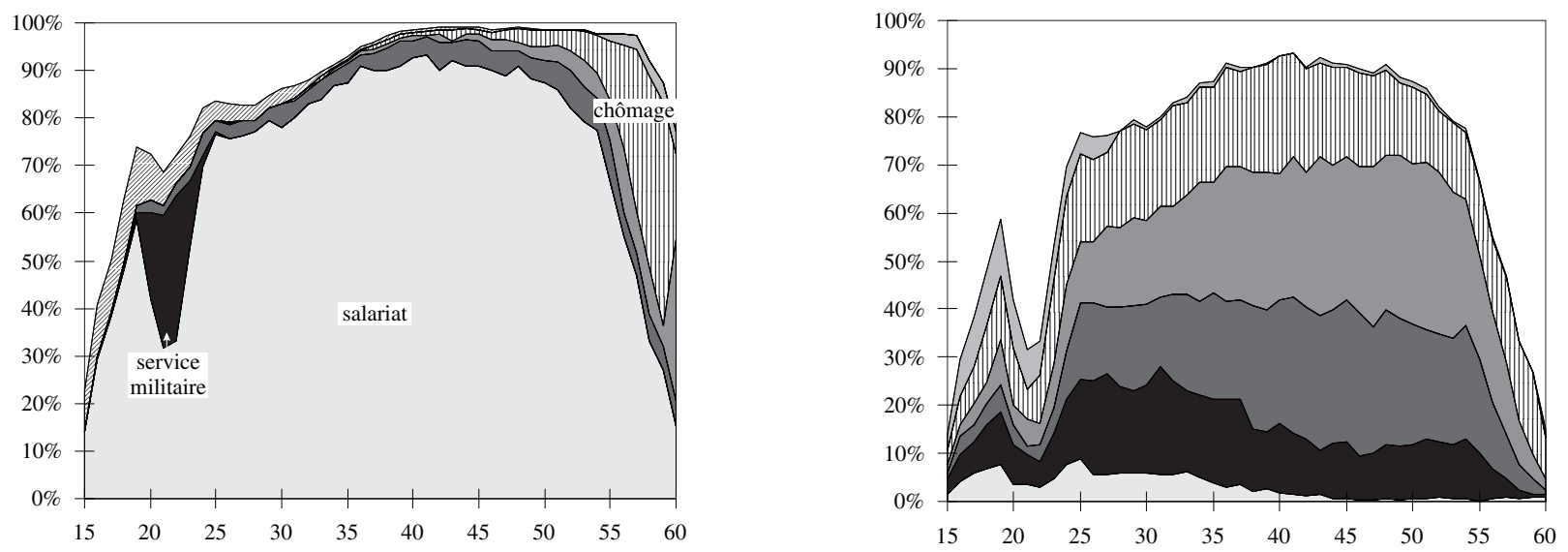

Groupe 7 : « longue et continue à salaires élevés » $(16 \%-23 \%)$
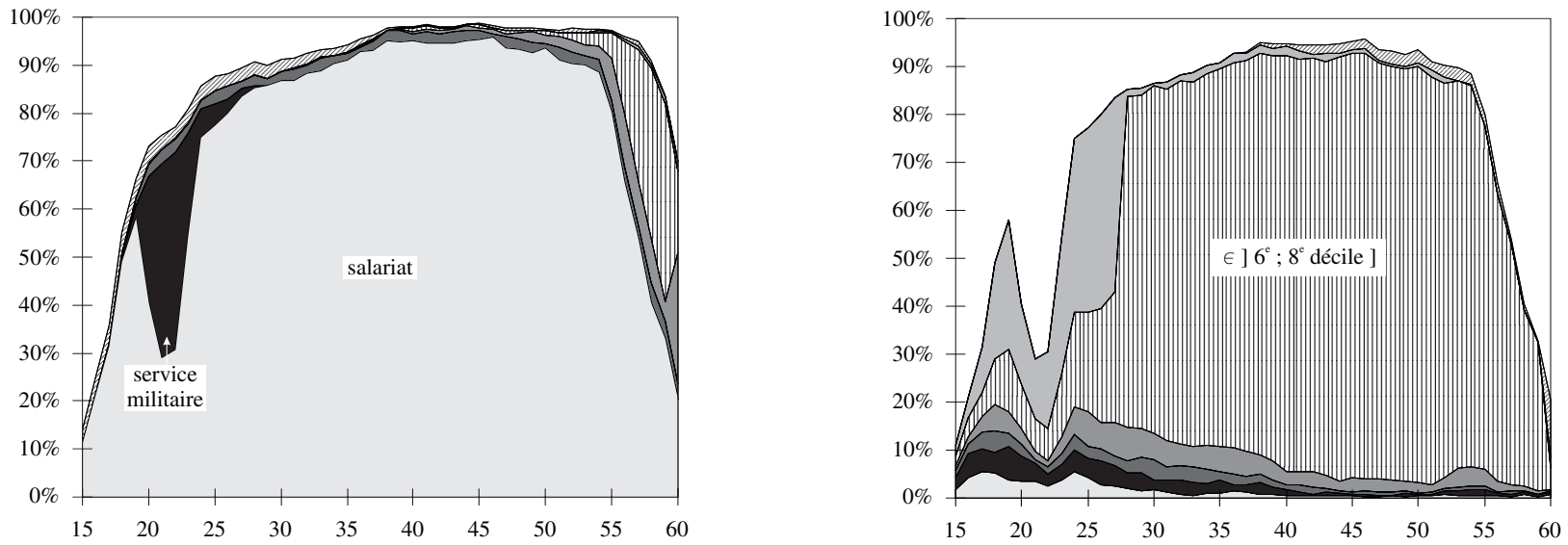
Figure 2 : composition de la carrière entre 15 et 60 ans des travailleurs passés par le secteur privé nés entre 1935 et 1940 (nombre moyen d'années)

Champ : 80427 assurés du Régime général nés entre 1935 et 1940 observés entre les âges de 15 et de 60 ans.

Le nombre d'années n'est mentionné que s'il est au moins égal à 2.

\section{L'évolution des carrières dans le secteur privé}

La classification des carrières des salariés du secteur privé nés entre 1935 et 1940 met en évidence des parcours professionnels divers, tant en termes de déroulement, de succession des différents états traversés (salariat, chômage, inactivité ...), que de progression salariale.

Si on se limite à la carrière avant 50 ans, il ressort que le parcours professionnel continu concerne plus de $60 \%$ de la population étudiée. Seuls suivent un parcours qui s'écarte de ce schéma les travailleurs devenus invalides (groupe 3) et les personnes retirées du marché du travail précocement et durablement (groupe 4) ou définitivement (la partie du groupe 1 non partie dans la fonction publique ou employée à l'étranger). En revanche, le parcours long et continu, entièrement effectué dans le secteur privé, est lui-déjà - minoritaire et ne représente que $46 \%$ des effectifs (groupes 5 à 7).

Les répercussions sur les parcours professionnels du ralentissement économique, de la montée de l'activité féminine et de l'émergence de formes d'emploi flexible des dernières décennies sont l'objet d'une interrogation qui peut se décliner en deux questions. La première est celle de la " déformation » des profils de carrière des salariés entrés sur le marché du travail à partir des années soixante par rapport à leurs aînés : dans quelle mesure la nature, la durée et l'ordre des différents épisodes qui composent leur parcours a t-elle changé ? La seconde question est celle de la modification de la «structure » du marché du travail : dans quelle proportion, les profils identifiés sur les générations 1935 à 1940 ont-ils persisté ?

La question de la déformation des profils de carrière peut être abordée en réalisant, génération par génération, une analyse de classification. Bien que l'on ne présente pas ici les groupes issus des analyses menées sur les générations 1941 à $1960^{(15)}$, il ressort que celles-ci identifient des profils de carrière similaires à ceux de la typologie mise en évidence sur les générations 1935 à 1940. Aucun nouveau profil n'émerge ou se substitue à un autre, de telle façon que la même grille d'analyse à sept parcours peut être

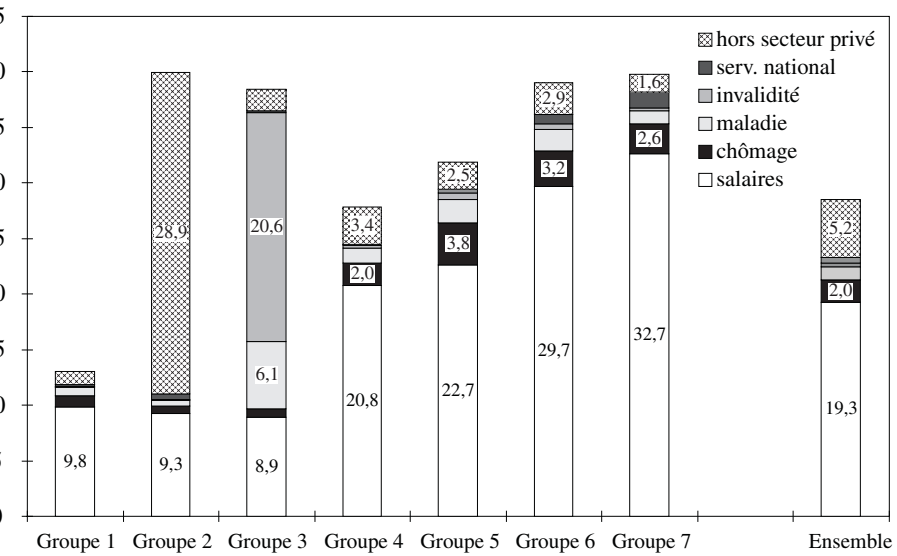

conservée. Seuls certains parcours comme ceux des groupes 4 et 5 se modifient et témoignent d'une plus longue présence sur le marché du travail, mais aussi d'une fréquence et d'une durée en situation de chômage accrues.

La question de l'évolution de la représentativité des profils de carrière des générations 1935 à 1940 aux générations 1941 à 1960 est abordée par une analyse discriminante : les sept groupes de la typologie identifiée sur les carrières, observées jusqu'à l'âge de 60 ans, des salariés nés entre 1935 et 1940 sont reliés aux carrières, observées entre 15 et 40 ans de 500000 assurés du Régime général nés entre 1935 et 1960. Si l'observation des 25 premières années de carrière permet de classer correctement les deux tiers des assurés des générations 1935 à $1940^{(16)}$, la procédure se voit néanmoins opposer deux limites.

- La première tient aux données : la connaissance tardive et partielle des trimestres validés hors du secteur privé conduit à affecter à tort des carrières du groupe 2 vers le groupe 1, particulièrement pour les salariés les plus jeunes.

- La seconde conséquence tient à l'approche adoptée : les groupes étant identifiés sur la seule période allant de 15 à 39 ans, tout changement intervenant à partir de l'âge de 40 ans est négligé ; en particulier, certains parcours professionnels à bas salaires et interrompus autour de 25 ans sont affectés au groupe 1 (carrière courte dans le secteur privé) et non au groupe 4 (carrière interrompue durablement à salaires bas) si une reprise d'activité dans le secteur privé intervient après 39 ans.

Il ressort (figure 3) que, quelles que soient les générations, les carrières continues à salaires proches du plafond (groupe 7) représentent une part constante de l'ordre de $20 \%$ de la population. Les carrières continues, mais moins bien rémunérées et aux évolutions salariales plus lentes du groupe 6, occupent quant à elles un poids croissant : $11 \%$ pour la génération 1935 contre $20 \%$ pour la génération 1960.

Cependant, cette évolution reste limitée au regard des transformations qui s'opèrent sur les segments 
Figure 3 : évolution de la structure du secteur privé

Champ : échantillon aléatoire de 500000 carrières d'assurés du Régime général des générations 1935 à 1960 observés jusqu'à 39 ans.

Lecture : $40 \%$ des assurés nés en 1935 appartiennent au groupe 1, $10 \%$ au groupe 2 , moins de $1 \%$ au groupe $3,10 \%$ au groupe $4,12 \%$ au groupe $5,11 \%$ au groupe 6 et $18 \%$ au groupe 7. Pour les assurés de la génération 1960, ces chiffres sont respectivement de $12 \%, 15 \%, 1 \%, 8 \%, 24 \%$, $20 \%$ et $21 \%$

aux carrières plus courtes, lesquels concernent plus des deux tiers des travailleurs passés par le secteur privé.

Parmi eux, une première catégorie réunit des carrières salariales continues et courtes : celles du groupe 1, avec sortie du marché du travail, transition vers la fonction publique ou départ à l'étranger avant 40 ans avec, dans certains cas, des retours dans le secteur privé après cet âge, et celles du groupe 2 avec sortie du secteur privé mais maintien en activité. La seconde catégorie rassemble les carrières des groupes 4 et 5 , marquées par des interruptions pour inactivité ou chômage et à salaires faibles. Alors que la part de la première diminue - elle représentait la moitié des assurés nés en 1935 et ne concerne plus que $26 \%$ de ceux nés en 1960 -, le poids de la seconde s'accroît, passant de $22 \%$ à $32 \%$. Cet effet de substitution tend à rendre compte de la multiplication des aléas de carrière sur les actifs jeunes (moins de 40 ans) et de son extension à des «types » de salariés jusqu'alors épargnés.

\section{Éléments de caractérisation des profils de carrière}

Dans leurs grandes tendances, les sept profils identifiés sur les carrières des salariés des générations 1935 à 1940 persistent sur les générations suivantes. Les parcours professionnels des assurés du Régime général nés entre 1935 et 1960 peuvent donc être appréhendés en retenant une typologie de carrières unique, « transgénérationnelle », où l'affectation à l'un des groupes est déterminée par les vingt-cinq premières années de carrière.

Le suivi sur plusieurs générations des caractéristiques de ces sept groupes de carrières, en termes de progressions salariales et d'exposition au chômage ${ }^{(17)}$, complétées de données sur la condition d'emploi, la catégorie sociale et le secteur d'activité, doit aider à préciser les différents profils de parcours - et d'assurés - identifiés et disposer alors d'une vision plus synthétique des changements qui ont pu affecter le secteur privé au cours des dernières décennies.

\section{Les carrières salariales}

Outre la nature et la succession des différents épisodes qui composent le parcours professionnel, le niveau et l'évolution des salaires apparaissent comme des éléments distinctifs des groupes de carrières mis au jour sur les générations 1935 à 1940.

Si à l'entrée sur le marché du travail les différences restent réduites, les écarts se creusent jusqu'à l'âge de 37 ans (figure 4). En moyenne, le salaire d'un assuré du groupe 7 né en 1938 est alors égal à $99 \%$ du plafond, celui d'un assuré du groupe 4 à $20 \%$. Les salaires sont maximaux entre 55 et 60 ans : 23065 euros 2000 en moyenne dans le groupe 7 à 57 ans et 9893 euros 2000 dans le groupe 4 à 56 ans.

Les différences de progression salariale entre les groupes distinguent, d'une part, des carrières discontinues, associées à des rémunérations peu élevées et de faibles progressions salariales et, d'autre part, des carrières continues avec une augmentation régulière des salaires. Cette dichotomie rejoint le schéma dual dérivé de la théorie du capital humain (Becker, 1975), selon lequel la stabilité dans l'emploi s'accompagne d'une acquisition continue de compétences alors que chaque interruption d'activité représente un arrêt dans la formation du capital humain et une dépréciation des savoirs et savoir-faire jusque-là acquis.

Pour prolonger 1'analyse de Doeringer et Piore (1971), les disparités de niveau et de progression salariale tiennent aussi au type d'emploi occupé dans le sens où les postes offrent des opportunités d'acquisition de capital humain différentes.

Si on considère désormais l'ensemble des salariés nés entre 1935 et 1960, dans les groupes 6 et 7, aux 
salaires en euros 2000

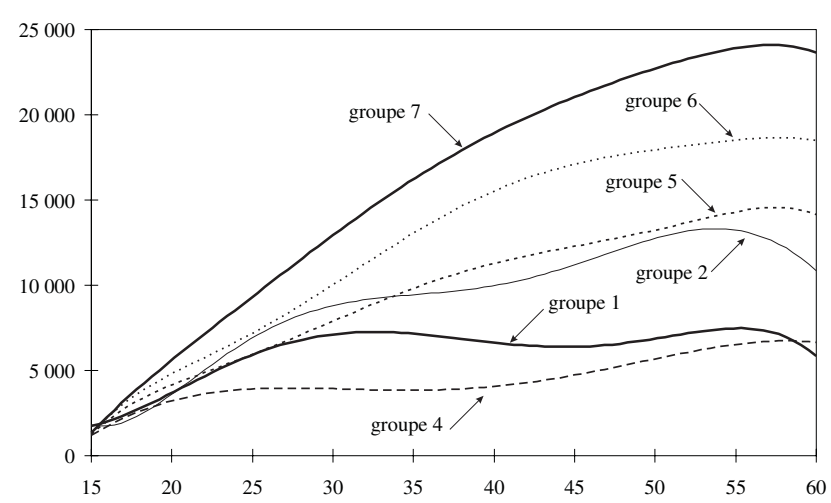

en $\%$ du plafond

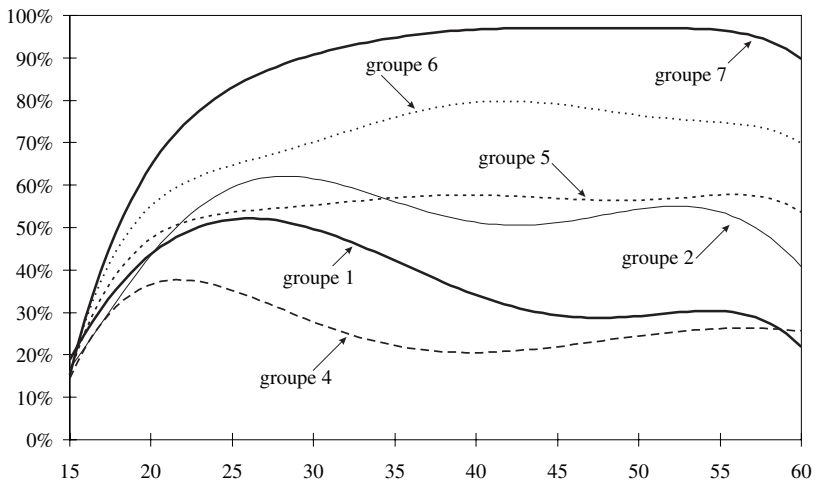

Champ : 80427 assurés du Régime général nés entre 1935 et 1940 observés entre 15 et 60 ans.

* En raison de la faiblesse des effectifs, le tracé pour le groupe 3 n'est pas reporté ; pour tous les groupes, il n'est pas significatif avant l'âge de 18 ans, car au plus le tiers des individus est salarié de façon régulière, de même que pour les groupes 1 et 2 au-delà de 25 et 28 ans respectivement.

carrières continues et longues, les emplois sont essentiellement à temps complet (figure 5). À l'opposé, les salariés aux carrières les plus courtes ou les plus discontinues occupent plus souvent des emplois dits «flexibles » : en 2000, un dixième des individus des groupes 1 à 5 travaillent par intermittence ${ }^{(18)}$ et au moins un cinquième à temps partiel. Ce type d'emplois est particulièrement fréquent dans les groupes 1 et 4 où, respectivement, $34 \%$ et $42 \%$ des salariés travaillent à temps partiel. Ces emplois à taux réduit, peu rémunérateurs, offrent des perspectives d'emploi limitées (Galtier, 1998), ce qui pourrait justifier que les assurés de ces groupes se retirent du marché du travail, temporairement voire définitivement.

Par ailleurs, ces chiffres sont vraisemblablement sous-estimés, car la condition d'emploi n'est connue que pour les deux tiers des salariés du groupe 4 , alors qu'elle est mentionnée pour $85 \%$ des assurés du Régime général qui ont été salariés dans le secteur

Figure 5 : condition d'emploi en 2000

Champ : salariés du secteur privé en emploi régulier en 2000 , issus d'un échantillon aléatoire de 500000 assurés du Régime général nés entre 1935 et 1960. privé au cours de l'année 2000 , pour $95 \%$ de ceux du groupe 7 , erreurs ou omissions de signalement, voire défaillances de la collecte des informations qui pourraient précisément être liées au caractère non «standard » de ces emplois ${ }^{(19)}$.

Les salariés nés dans les années quarante entrent sur le marché du travail dans un contexte économique favorable où l' emploi à vie est la norme et le retrait du marché du travail résulte d'un choix non contraint par les circonstances économiques. Leurs cadets font leur entrée dans la vie active alors que la conjoncture se fait plus morose, dans un contexte de mutations technologiques rapides, où les débouchés se raréfient, particulièrement pour les moins qualifiés qui peuvent multiplier les emplois précaires, les périodes de chômage voire d'inactivité, et ne peuvent plus s'attendre qu'à une progression limitée de leurs rémunérations.

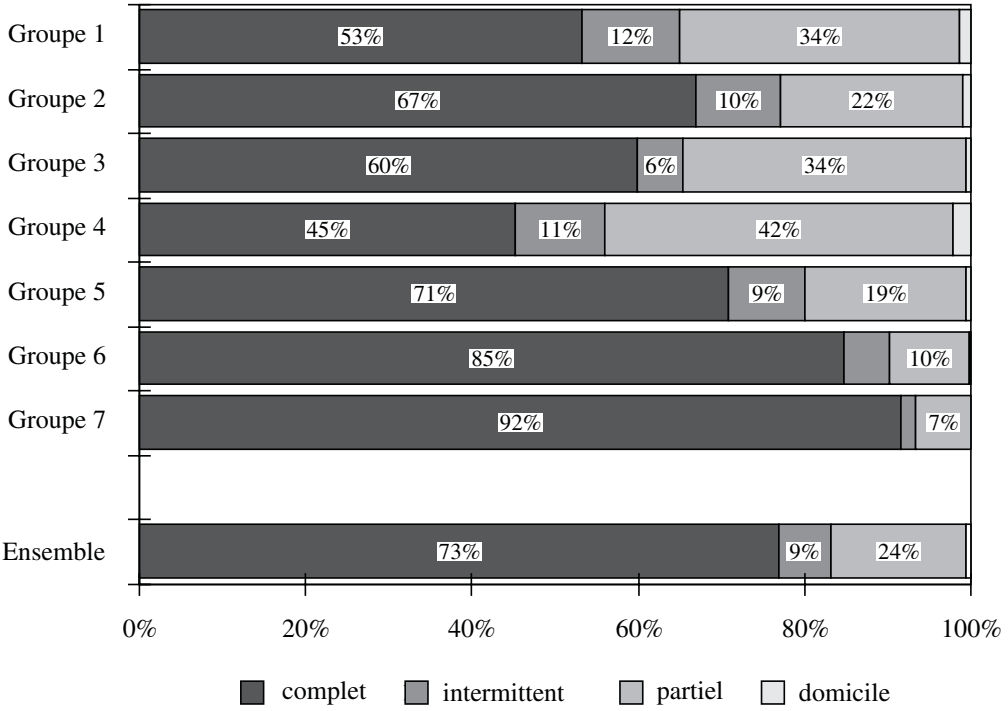


Lollivier et Payen (1990) montrent ainsi que les écarts de progression salariale les plus nets apparaissent entre les générations nées avant le baby boom (1935 à 1945) et celles de la seconde moitié des années cinquante. De la même façon, Koubi (2004) montre que, toutes choses égales par ailleurs, le taux de croissance des salaires entre 30 et 50 ans est supérieur de 5,3\% pour les salariés nés en 1942 par rapport à celui des salariés nés en 1950, le différentiel se réduisant de façon continue entre ces deux générations ${ }^{(20)}$. Dans le même temps, l'âge auquel la rémunération est la plus élevée augmente, passant de 40 ans pour les hommes en 1970 à plus de 45 ans en 1993 (Baudelot et Gollac, 1997) ; par conséquent, l'espérance de gains à 40 ans se réduit.

Néanmoins, si le rythme de croissance des salaires est différent selon le type de carrière suivie (figure 6), le ralentissement de la progression salariale se concentre aussi sur les carrières les plus discontinues et les moins rémunérées : des générations 1935 à 1940 aux générations 1956 à 1960, alors qu'entre 20 et 40 ans, le taux de croissance annuel des salaires se maintient dans le groupe 7, il baisse de l'ordre de 2 points dans les groupes $1,2,4$ et 6 , et de 2,4 points dans le groupe 5 en passant de $5,4 \%$ à 3,0\% .

En outre, la difficulté croissante à occuper un emploi stable se manifeste par une diminution de la proportion d'assurés du Régime général qui exerce une activité régulière dans le secteur privé 8 . Cette baisse se concentre là encore sur le groupe 5 dont la proportion à l'âge de 40 ans passe de $74 \%$ pour les générations 1935 à 1940 à $50 \%$ pour les générations 1956 à 1960 (figure 7), mais n'épargne pas le groupe 6 , pour lequel la proportion passe de $88 \%$ à $70 \%$.

\section{Le chômage : risque et durée}

La probabilité de perdre son emploi s'est accrue tendanciellement au cours des vingt dernières années, ce qui s'est traduit par des périodes de chômage de plus en plus tôt dans la vie active. À 40 ans, la proportion d'assurés du Régime général validant des trimestres d'assurance au titre du

Figure 6 : taux annuel moyen de croissance des salaires entre 20 et 40 ans

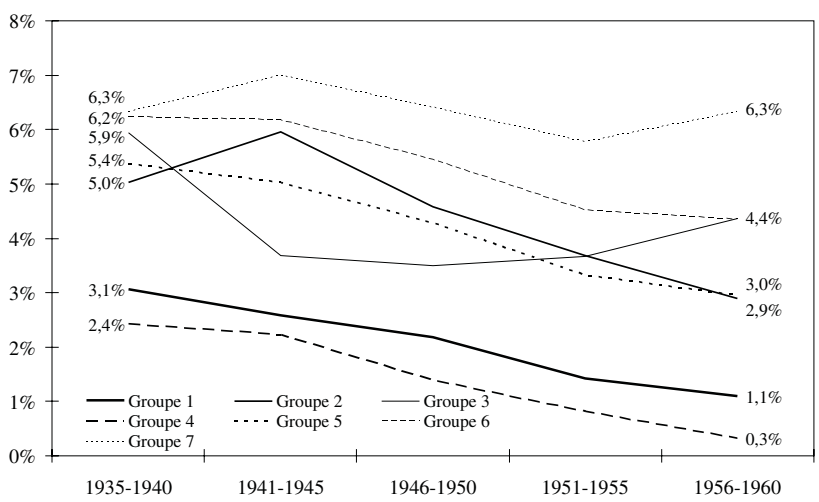

chômage est passée de 1,9 \% pour ceux nés entre 1935 à 1940 à $11,8 \%$ pour ceux nés entre 1956 et 1960 (tableau 2). Cela correspond à un rythme moyen de croissance de l'ordre de $10 \%$ par an, soit plus de deux fois supérieur à l'augmentation du taux de chômage de la classe d'âges 25-49 ans, lequel est passé de 2,9\% à 8,9\% sur la période 1975-2000 (Insee, Enquêtes Emploi).

Cet écart trouve deux explications : d'une part, le chômage se concentre sur le secteur privé alors que les autres secteurs de l'économie sont relativement épargnés ; d'autre part, les périodes de chômage figurent au compte individuel de l'assuré du Régime général lorsqu'elles donnent droit à la validation d'un trimestre d'assurance (une «période assimilée» ou PA), c'est-à-dire à partir de 50 jours d'indemnisation dans l'année civile à condition que l'assuré soit préalablement affilié. Entre 1975 et 2000, la durée moyenne du chômage fait plus que doubler en passant de 8 à près de 17 mois (Insee), avec pour conséquence une augmentation du nombre de travailleurs dont la période de chômage excède 50 jours et une augmentation du nombre de trimestres validés au titre du chômage. En moyenne, l'année de son $40^{\mathrm{e}}$ anniversaire, un assuré né entre 1935 et 1940 a ainsi validé 2,77 PA-chômage alors qu'un assuré né entre 1956 et 1960 en a validé 4,24 (tableau 3).

Que l'on considère la proportion de personnes validant des périodes de chômage ou le nombre moyen de trimestres d'assurance validés, le groupe 5 reste le plus touché : pour les générations 1935 à 1940, trois fois plus d'individus sont concernés que dans le reste de la population $(6,0 \%$ contre $1,9 \%$; tableau 2), pour les générations 1956 à 1960, au moins deux fois plus $(26,6 \%$ contre $11,8 \%)$ et, lorsqu'ils sont au chômage, les assurés du groupe 5 valident en moyenne 1,15 fois plus de trimestres d'assurance au titre du chômage qu'un assuré « moyen » $(4,78$ contre 4,24 pour les générations 1956 à 1960 par exemple; tableau 3$)^{(21)}$.

Figure 7 : taux de report de salaire à 40 ans

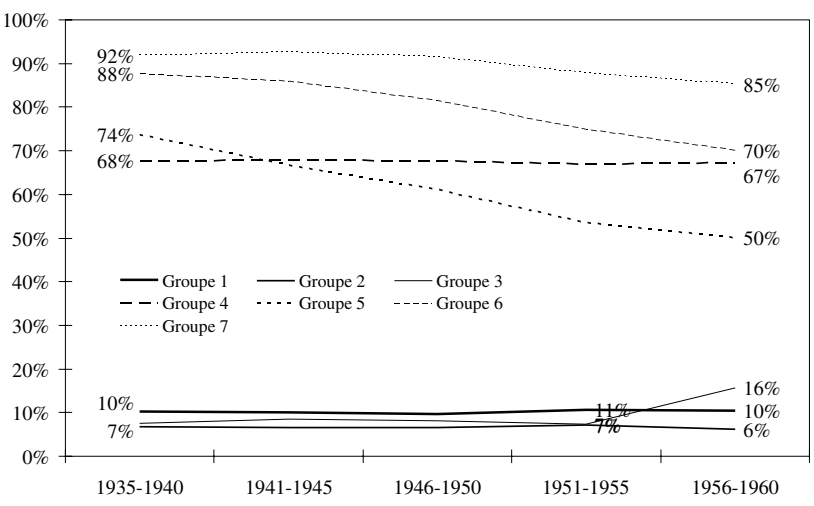


Cette plus grande exposition au chômage peut être rapprochée de la faible progression salariale déjà observée pour ce groupe : les entreprises en difficulté contiennent l'augmentation de leur masse salariale avant de procéder à des licenciements et, comme chaque interruption de carrière constitue une rupture dans l'acquisition de compétences et envoie un signal négatif en direction d'employeurs potentiels, on observe une corrélation négative entre la fréquence et/ou la durée du chômage et le salaire à l'embauche.

Cette vulnérabilité au chômage des salariés du groupe 5 semble les distinguer des salariés du groupe 4. Ceux-ci ont des profils d'activité proches, des salaires faibles, mais leurs interruptions de carrière, plus durables, pourraient davantage être le résultat

Figure 8 : proportion d'assurés validant au moins une période de chômage à $40,45,50$ et 55 ans générations 1935 à 1940

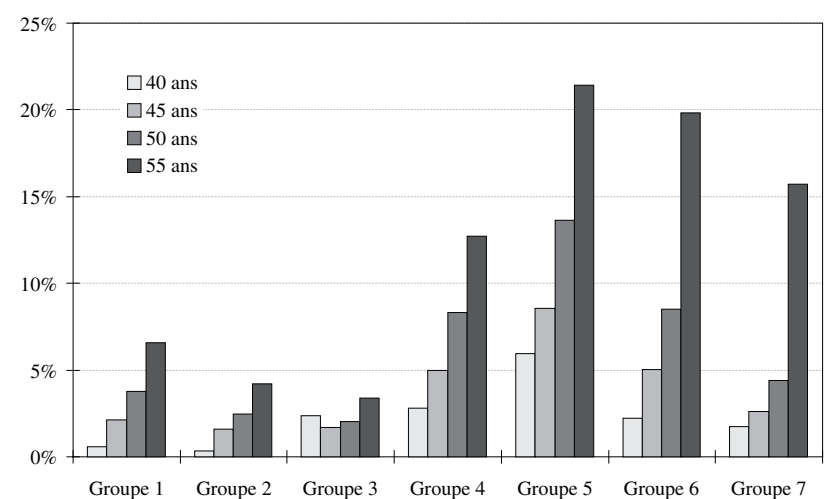

Champ : 80427 assurés du Régime général nés entre 1935 et 1940. d'un choix personnel de travailler par intermittence pour accorder plus de temps à des activités domestiques ou de loisir, ou de se retirer temporairement du marché du travail au vu de perspectives professionnelles limitées.

Aucune des catégories d'assurés identifiées n'est cependant protégée vis-à-vis du chômage. Même si, parmi les assurés nés entre 1935 et 1940, ceux du groupe 5 sont toujours les plus concernés par ce chômage de fin de carrière (dès 40 ans, ils sont déjà $6 \%$ à valider des PA-chômage et sont $21 \%$ à 55 ans), $20 \%$ des assurés du groupe 6 et $16 \%$ du groupe 7 , aux carrières longues et continues, valident des périodes de chômage l'année de leurs 55 ans (figure 8 ) et ces proportions augmentent jusqu'à l'âge de 60 ans. De nombreux individus épargnés durant toute leur carrière achèvent ainsi leur vie active sans emploi.

\section{Les caractéristiques socio-économiques des salariés passés par le secteur privé}

Catégorie sociale et secteur d'activité sont souvent retenus comme facteurs explicatifs de la dispersion des salaires et, plus généralement, de l'hétérogénéité du marché du travail ${ }^{(22)}$. En particulier, à défaut de disposer des éléments centraux de la théorie du capital humain que sont le niveau d'études et l'ancienneté dans l'emploi, les travaux menés sur les DADS retiennent la catégorie sociale comme indicateur de qualification. Les approches plus centrées sur la demande de travail font en outre exercer une influence particulière aux modes de gestion de la main-d'œuvre et aux technologies de production, et privilégient alors des stratifications selon le secteur d'activité.

Tableau 2 : proportion d'assurés validant au moins une période de chômage l'année de leurs 40 ans (en \%)

\begin{tabular}{|c|ccccccc|c|}
\hline Générations & \multicolumn{9}{c}{ Groupes } & 6 & \multicolumn{2}{c|}{ Ensemble } \\
\hline $1935-1940$ & 1 & 2 & 3 & 4 & 5 & 6,2 & 1,7 & 1,9 \\
$1941-1945$ & 1,4 & 0,4 & 2,4 & 2,8 & 6,9 & 2,2 & 4,4 \\
$1946-1950$ & 2,1 & 1,6 & 3,4 & 8,0 & 21,0 & 8,5 & 3,3 & 7,1 \\
$1951-1955$ & 2,5 & 1,1 & 4,6 & 8,9 & 26,8 & 14,3 & 5,6 & 10,5 \\
$1956-1960$ & 2,3 & 1,2 & 7,1 & 8,6 & 26,6 & 16,5 & 5,6 & 11,8 \\
\hline
\end{tabular}

Champ : 500000 assurés du Régime général des générations 1935 à 1960.

Tableau 3 : nombre moyen de trimestres de chômage validés l'année des 40 ans

\begin{tabular}{|c|c|c|c|c|c|c|c|c|}
\hline \multirow{2}{*}{ Générations } & \multicolumn{7}{|c|}{ Groupes } & \multirow{2}{*}{ Ensemble } \\
\hline & 1 & 2 & 3 & 4 & 5 & 6 & 7 & \\
\hline $1935-1940$ & 2,77 & 2,41 & 1,74 & 2,82 & 3,14 & 2,82 & 2,68 & 2,77 \\
\hline $1941-1945$ & 3,38 & 3,08 & 1,44 & 3,77 & 4,01 & 3,47 & 3,28 & 3,46 \\
\hline $1946-1950$ & 3,52 & 3,38 & 3,35 & 4,05 & 4,59 & 4,25 & 4,07 & 3,96 \\
\hline $1951-1955$ & 3,73 & 3,30 & 6,02 & 4,56 & 4,99 & 4,57 & 4,6 & 4,37 \\
\hline $1956-1960$ & 3,79 & 3,67 & 4,25 & 4,17 & 4,78 & 4,34 & 4,21 & 4,24 \\
\hline
\end{tabular}

Champ : assurés du Régime général nés entre 1935 et 1960, ayant validé au moins un trimestre d'assurance au titre du chômage l'année de leurs 40 ans. 
Les fichiers de la Cnav ne mentionnent ces informations qu'à compter de 1999, ce qui empêche tout suivi longitudinal et contraint à étudier simultanément plusieurs classes d'âges pour disposer d'effectifs suffisants. Or l'observation de la catégorie sociale et du secteur d'activité à une date donnée et non sur plusieurs années, rend difficile le contrôle des effets d'âge et de date.

Certes, la mobilité intersectorielle se restreint le plus souvent à des activités où les modes de gestion de la main d'œuvre sont voisins (Le Minez, 2002) et les effets d'âge expliquent donc vraisemblablement peu la diversité des secteurs occupés par les travailleurs d'un même groupe, cela d'autant plus que l'on s'intéresse à des travailleurs qui entrent dans la seconde moitié de leur carrière. En revanche, l'observation de la catégorie socioprofessionnelle à une date donnée reste un indicateur fruste : la coexistence de différentes PCS au sein d'un même groupe peut autant rendre compte d'un effet de structure lié à la progression sociale des travailleurs avec l'ancienneté, qu'à une fragilité de la relation entre la catégorie socioprofessionnelle et le niveau de rémunération. Celle-ci a d'ailleurs été justifiée par une évolution du contenu des emplois et un phénomène de déclassement conjoncturel durant les années quatre-vingt et quatre-vingt-dix, les compétences requises à l'embauche étant généralement supérieures aux compétences nécessaires pour l'emploi (Forgeot et Gautié, 1997; Ponthieux, 1997; Nauze-Fichet et Tomasini, 2002).

En dépit de ces limites, quelques éléments peuvent être mis en évidence.

En 2001, parmi les salariés du secteur privéâgés d'au moins 40 ans, en emploi, moins de $1 \%$ travaillent dans l'Agriculture, $21 \%$ dans l'Industrie, $6 \%$ dans le secteur de la construction et $72 \%$ dans le Tertiaire (tableau 4) ${ }^{(23)}$. Cette répartition est relativement uniforme entre les différentes classes d'âges observées (figure 9). Seuls les salariés de plus de 60 ans (générations 1935 à 1940) travaillent plus fréquemment dans le secteur tertiaire $(82 \%)$ et comptent également davantage de cadres $(21 \%)$ et d'employés (48\%). Ces catégories sont en effet moins concernées par les dispositifs de cessation anticipée d'activité qui sont avant tout destinés à une main d'œuvre ouvrière dans l'Industrie (Colin et alii, 2000 ; Cloarec, 2000) $)^{(24)}$.

Avec l'âge, la proportion de salariés exerçant une profession intermédiaire décroît alors qu'augmente celle des cadres, ces derniers ne représentant que $13 \%$ des salariés âgés de 41 à 45 ans (figure 9). Cette évolution est pour une large part imputable au mécanisme de promotion sociale, mais l'effet de ciseaux, caractérisé par un poids croissant des employés et un poids décroissant des ouvriers, est lui essentiellement attribuable au processus de tertiarisation et au recul de l'Industrie depuis la moitié des années soixante-dix.

Les fichiers de la Cnav ne permettent pas de distinguer les périodes de préretraite des situations de chômage ou d'inactivité. Il apparaît cependant que les assurés des groupes 6 et 7, dont les taux de salariat chutent à partir de 55 ans (figure 2), sont largement présents dans l'Industrie. Ils en représentent $58 \%$ des effectifs et plus du tiers des individus de ces groupes travaille dans ce secteur (33\% pour le groupe $6,36 \%$ pour le groupe 7 ; tableau 4).

Les cadres et professions intermédiaires sont au moins deux fois plus présents dans le groupe 7 que dans l'ensemble de la population et près de la moitié des effectifs de ces catégories appartient à ce groupe (tableau 5). Les travailleurs des groupes 5 et 6

Figure 9 : PCS et secteurs d'activité par génération en 2001
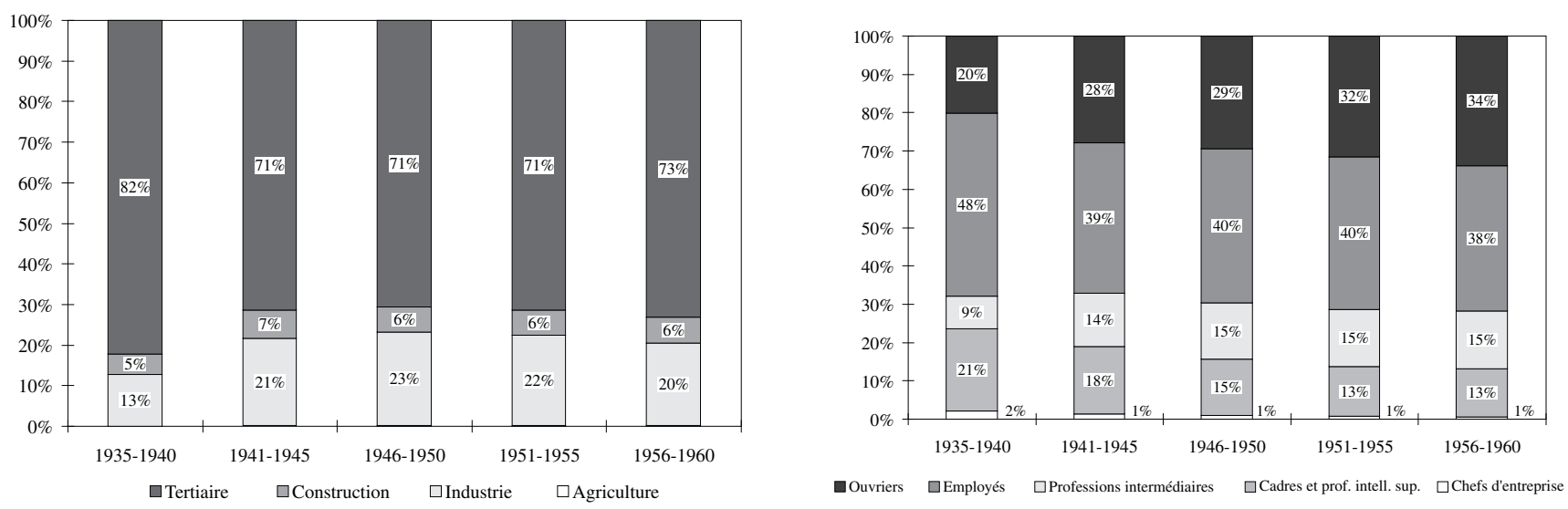

Champ : 500000 assurés du Régime général nés entre 1935 et 1960, salariés dans le secteur privé en 2001.

Lecture : 73 \% des salariés en 2001 nés entre 1956 et 1960 (âgés alors de 41 à 45 ans) travaillaient dans le Tertiaire. 
occupent des catégories sociales plus diverses. Ils sont cependant d'autant plus présents que l'on descend l'échelle sociale : le groupe 6 compte $6 \%$ de cadres, $14 \%$ de professions intermédiaires, $27 \%$ d'employés et plus de la moitié des effectifs est ouvrière ; dans le groupe 5 , ces parts s'établissent respectivement à $6 \%, 8 \%, 41 \%$ et $44 \%$. Près de $80 \%$ des ouvriers du groupe 6 sont des ouvriers qualifiés, alors qu'ils sont moins de $60 \%$ dans le groupe 5 .

Plus de la moitié des personnes qui travaillent dans le secteur de la construction se retrouve dans ces deux groupes. Le secteur est sous-représenté dans le groupe 4, majoritairement féminin. En revanche, $87 \%$ des carrières du groupe 4 sont suivies dans le Tertiaire, près des deux tiers des salariés sont des employés et, parmi les $22 \%$ d'ouvriers, plus de $70 \%$ sont non qualifiés.
De façon générale, le lien entre la catégorie sociale et le salaire est préservé par la typologie, les carrières des groupes où les cadres sont majoritaires sont mieux rémunérées que celles des groupes composés essentiellement d'employés et d'ouvriers.

La surreprésentation des employés et ouvriers dans des groupes aux carrières discontinues, tels que les groupes 4 et 5 , pourrait aussi être associée à leur plus grande mobilité. Ces catégories sociales sont en effet deux à trois fois plus nombreuses à changer d'entreprise au cours des trente premières années de carrière (Amossé, 2003) et les moins qualifiés connaissent également plus fréquemment le chômage ou l'inactivité (Bayet, 1996a ; Chardon, 2002 ; Germe et alii, 2003).

Tableau 4 : répartition par secteurs d'activité

\begin{tabular}{|c|c|c|c|c|c|c|c|c|}
\hline \multirow{2}{*}{ en $\%$} & \multicolumn{7}{|c|}{ Groupes } & \multirow{2}{*}{ Ensemble } \\
\hline & 1 & 2 & 3 & 4 & 5 & 6 & 7 & \\
\hline Agriculture & 0 & 0 & 0 & 0 & 0 & 0 & 0 & 0 \\
\hline Industrie & 10 & 15 & 20 & 9 & 22 & 33 & 36 & 21 \\
\hline Construction & 4 & 14 & 5 & 3 & 10 & 10 & 3 & 6 \\
\hline Tertiaire & 86 & 71 & 76 & 87 & 68 & 57 & 61 & 72 \\
\hline Ensemble & 100 & 100 & 100 & 100 & 100 & 100 & 100 & 100 \\
\hline
\end{tabular}

\begin{tabular}{|l|cccccccc|}
\hline \multicolumn{1}{c|}{ en \% } & \multicolumn{9}{c}{ Groupes } & 5 & 6 & 7 & Ensemble \\
\cline { 2 - 9 } Agriculture & 1 & 2 & 3 & 4 & 16 & 6 & 3 & 100 \\
Industrie & 45 & 7 & 0 & 23 & 19 & 24 & 34 & 100 \\
Construction & 14 & 5 & 0 & 4 & 29 & 24 & 11 & 100 \\
Tertiaire & 17 & 15 & 0 & 5 & 18 & 12 & 17 & 100 \\
\hline Ensemble & 35 & 7 & 0 & 12 & 19 & 15 & 20 & 100 \\
\hline
\end{tabular}

Champ : 500000 assurés du Régime général nés entre 1935 et 1960, salariés dans le secteur privé en 2001 ; effectifs pondérés de façon à conserver la représentativité de chaque groupe.

Tableau 5 : répartition par professions et catégories socioprofessionnelles

\begin{tabular}{|c|c|c|c|c|c|c|c|c|}
\hline \multirow{2}{*}{ en $\%$} & \multicolumn{7}{|c|}{ Groupes } & \multirow{2}{*}{ Ensemble } \\
\hline & 1 & 2 & 3 & 4 & 5 & 6 & 7 & \\
\hline Chefs d'entreprise & 1 & 3 & 0 & 0 & 0 & 0 & 2 & 1 \\
\hline Cadres et prof. intell. sup. & 11 & 21 & 10 & 6 & 6 & 6 & 35 & 14 \\
\hline Professions intermédiaires & 10 & 13 & 14 & 6 & 8 & 14 & 31 & 15 \\
\hline Employés & 58 & 37 & 39 & 65 & 41 & 27 & 13 & 40 \\
\hline Ouvriers qualifiés & 8 & 20 & 15 & 6 & 26 & 41 & 18 & 21 \\
\hline Ouvriers non qualifiés & 12 & 7 & 21 & 16 & 18 & 12 & 2 & 10 \\
\hline Ensemble & 100 & 100 & 100 & 100 & 100 & 100 & 100 & 100 \\
\hline
\end{tabular}

\begin{tabular}{|l|ccccccc|c|}
\hline \multicolumn{1}{c|}{ en \% } & \multicolumn{7}{c}{ Groupes } & \multicolumn{2}{c|}{ Ensemble } \\
\cline { 2 - 9 } Chefs d'entreprise & 1 & 2 & 3 & 4 & 6 & 6 & 38 & 100 \\
Cadres et prof. intell. sup. & 27 & 20 & 0 & 3 & 8 & 6 & 49 & 100 \\
Professions intermédiaires & 22 & 10 & 0 & 4 & 10 & 15 & 44 & 100 \\
Employés & 21 & 6 & 0 & 4 & 19 & 10 & 6 & 100 \\
Ouvriers qualifiés & 43 & 6 & 0 & 15 & 23 & 30 & 17 & 100 \\
Ouvriers non qualifiés & 12 & 6 & 0 & 3 & 36 & 19 & 4 & 100 \\
Ensemble & 37 & 5 & 1 & 16 & 19 & 15 & 20 & 100 \\
\hline
\end{tabular}

Champ : 500000 assurés du Régime général nés entre 1935 et 1960, salariés dans le secteur privé en 2001 ; effectifs pondérés de façon à conserver la représentativité de chaque groupe. 
Le secteur d'activité permet également de justifier une partie des différences de rémunérations. Ainsi, selon la théorie du dualisme du marché du travail, le secteur primaire concerne essentiellement une industrie intensive en capital, à productivité du travail élevée, alors que le secteur secondaire est constitué d'entreprises recourant à une main-d'œuvre peu qualifiée et présentant un taux de rotation soutenu. Aussi, ces deux secteurs opposent-ils des travailleurs ayant un emploi stable, des rémunérations élevées et qui croissent de façon continue, à des travailleurs changeant fréquemment d'employeur et qui ont peu de possibilités de valoriser leur expérience professionnelle. Cette analyse a souvent été reprise afin de mettre en évidence la différenciation des trajectoires salariales entre les secteurs d'activité (cf. entre autres Osberg et alii, 1987) et cela bien que l'imperfection d'une telle stratification ait de nombreuses fois été soulignée, plusieurs types d'emplois et de systèmes de rémunération pouvant coexister au sein d'une même entreprise (Doeringer et Piore, 1971 ; Dickens et Lang, 1985).

Araï et alii (1996) estiment que l'appartenance au secteur d'activité explique à elle seule de $6 \%$ à $17 \%$ de la variance des salaires individuels ${ }^{(25)}$. Ces différentiels sectoriels de salaire peuvent résulter d'un mécanisme de partage des bénéfices entre entreprise et employés (rent sharing) - les entreprises de production et de distribution d'énergie, en situation de quasi-monopole, en constituant à ce titre un exemple manifeste - ou, plus généralement, de la nature des processus de production mis en œuvre. Une différence s'opère ainsi entre, d'une part, les secteurs à forte intensité capitalistique, comme l'Énergie, l'industrie des biens intermédiaires, des transports et, dans une moindre mesure, des industries agroalimentaires, où la productivité du travail et les frais de personnel sont élevés, la main-d'œuvre qualifiée ; et, d'autre part, des secteurs, comme l'Industrie des biens de consommation et le Commerce, qui utilisent davantage le facteur travail (Lainé, 1999).

Il ressort que les individus des groupes 4 et 5 sont sous-représentés dans les secteurs les plus rémunérateurs (énergie, activités financières, industries des biens d'équipement et de biens intermédiaires) et davantage présents dans des secteurs de «main-d'œuvre», aux salaires relativement peu élevés, tels que l'Industrie

\section{Figure 10 : différentiels sectoriels de salaire et représentativité des groupes}

Groupes 1 et 4

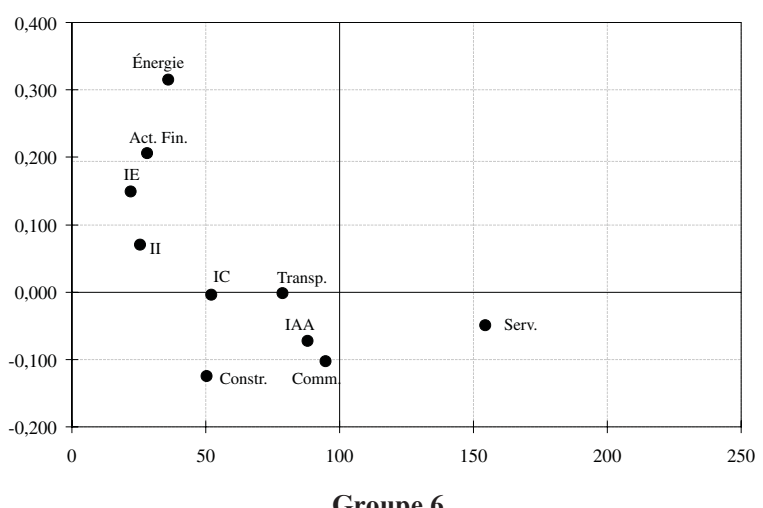

Groupe 6

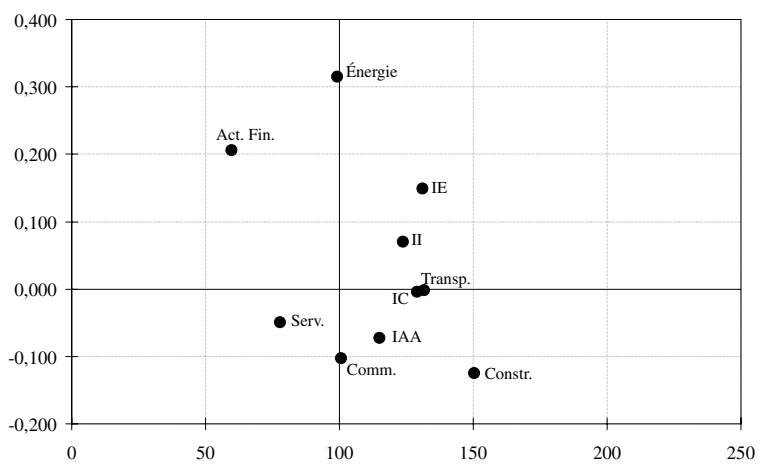

Groupe 5
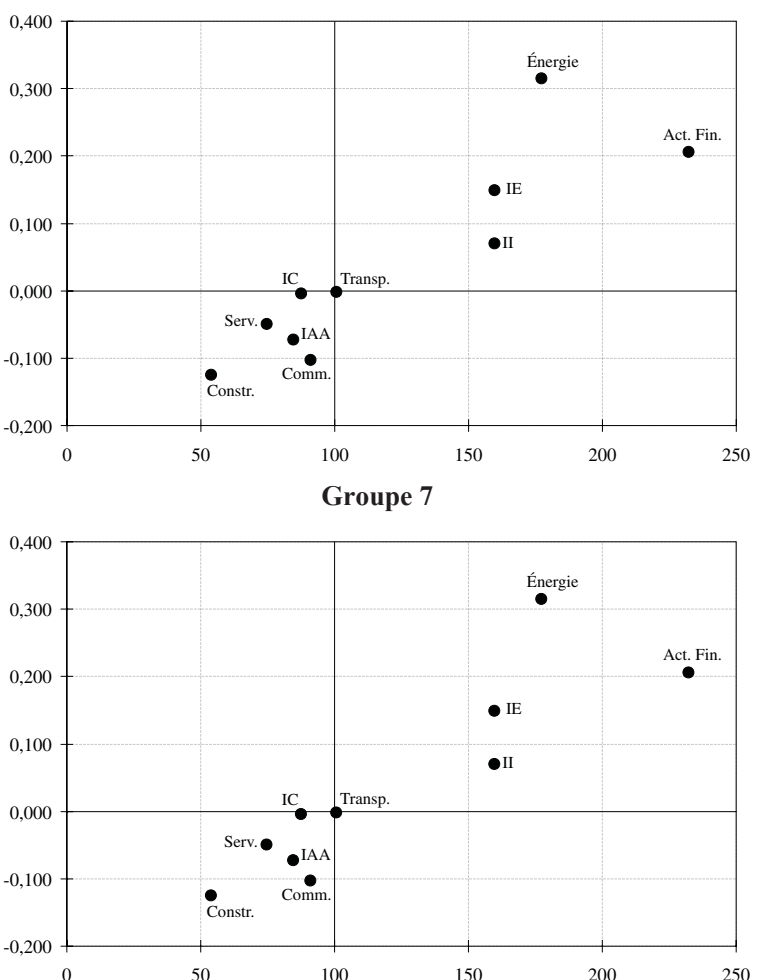

En ordonnées : différentiels de salaire entre le travailleur moyen du secteur et le travailleur moyen de l'économie sans tenir compte des caractéristiques individuelles (Araï et al., 1996)

En abscisses : représentativité du secteur dans le groupe relativement à sa représentativité tous groupes confondus ; champ : 500 000 assurés du Régime général nés entre 1935 et 1960, salariés dans le secteur privé en 2001.

10 secteurs : IAA - Industries agroalimentaires, Energie, II - Industries de biens intermédiaires, IE - Industries de biens d'équipement, IC - Industries des biens de consommation courante, Constr - Construction, Comm - Commerce, Transp - Transports et communications, Serv - Services, Act.Fin. Activités financières.

On ne présente pas ici les graphes relatifs aux groupes 2 et 3 pour lesquels le salaire n'est pas apparu comme un élément distinctif et les résultats très voisins des groupes 1 et 4 nous amènent à les représenter sur le même graphe. 
agroalimentaire ou le Commerce, avec une surreprésentation nette du groupe 4 dans les Services et du groupe 5 dans le secteur de la construction (figure 10). À l'opposé, les travailleurs des groupes 7 et 8 se situent essentiellement dans les secteurs aux salaires les plus élevés (activités financières, Énergie, IE et II) et tendent à être moins présents dans les secteurs tels que la Construction ou le Commerce. Enfin, le groupe 6 occupe une position intermédiaire : il compte davantage de travailleurs dans les industries de l'équipement et des biens intermédiaires, mais également dans les secteurs les moins rémunérateurs comme la Construction.

Aussi fines que soient les nomenclatures adoptées, les données ne permettent pas d'identifier avec précision la nature des emplois occupés et les technologies de production. Et bien que des proximités se dégagent de façon nette, la distension du lien entre rémunération d'une part, catégories sociales et secteur d'activité d'autre part, se traduit par une impossibilité de transposer la typologie de carrières des salariés du secteur privé en une stratification par catégories sociales ou secteurs d'activité. Comme l'a noté Gautié (2003), « au-delà des différences inter-catégorielles, c'est au sein même de chaque catégorie (professionnelle mais aussi d'âge, de diplôme, etc.) que semblent se différencier les trajectoires ».

\section{Modification des trajectoires dans le secteur privé et transformations du marché du travail}

La mise en évidence de régularités dans les parcours professionnels des salariés du secteur privé et le suivi de leurs caractéristiques sur les générations 1935 à 1960 permettent d'apporter un éclairage sur l'amplitude et la nature de la remise en cause de la carrière continue au cours des dernières décennies.

Sans étudier les parcours suivis par les premières générations de salariés affiliés au Régime général, il reste difficile de dire si le modèle de la carrière continue de la sortie des études à la retraite a jamais été dominant. Les parcours suivis par les assurés nés entre 1935 et 1940 ne répondaient déjà plus à ce schéma, mais ils n'en déviaient qu'à l'approche de la retraite. On constate néanmoins que, si pour les générations actuellement retraitées, les « carrières continues dans le secteur privé » rassemblaient près de $50 \%$ des effectifs (groupes 5 à 7), pour les travailleurs nés en 1960, elles n'en représentaient plus qu'un tiers (groupe 7, voire groupe 6), laissant ainsi persister le modèle historique, mais affaiblissant durement sa représentativité.

Dans cette évolution, deux grands traits ressortent : une précarisation de certains parcours et l'extension de cette fragilisation.

Les deux dernières décennies ont été marquées par l'accroissement des déséquilibres sur le marché du travail avec une augmentation quasi continue du nombre de demandeurs d'emploi et de la proportion des emplois faiblement rémunérés. Entre 1983 et 1997, la part des bas salaires (au plus égaux aux deux tiers du salaire médian) est passée de $11,4 \%$ à $15,1 \%$, celle des très bas salaires (au plus égaux à la moitié du salaire médian) de 5,0\% à 10,1\% (Concialdi et Ponthieux, 1999). Un des signes en est le poids croissant des carrières présentant des périodes de chômage et d'inactivité, et dont les salaires sont majoritairement inférieurs au salaire médian (plafonné) à partir de l'âge de 30 ans (groupe 5).

Dans le même temps, la durée passée sur le marché du travail - non nécessairement en emploi s'allonge; en témoigne la baisse de la proportion de parcours marqués par une sortie définitive du secteur privé (groupes 1 et 2), au profit de parcours plus longs, mais interrompus plus fréquemment (groupes 4 et 5).

Les explications qui peuvent être avancées sont nombreuses. L'affaiblissement des solidarités familiales et la multiplication des personnes vivant seules ont vraisemblablement contraint plus d'individus à se présenter et à rester sur le marché du travail pour garantir la continuité de leurs ressources. Les travailleurs immigrés, venus à partir de 1954 pour pallier la pénurie de main-d'œuvre ouvrière et retournés dans leur pays d'origine ${ }^{(26)}$, ont laissé place à une main d'œuvre sédentaire qui s'est trouvée confrontée à des difficultés croissantes sur le marché du travail. Le ralentissement des recrutements dans le secteur public, après de nombreuses créations d'emploi dans les années soixante-dix, pourrait aussi justifier la baisse de la proportion des carrières les plus courtes.

De façon plus évidente, cet allongement de la durée passée dans le secteur privé renvoie aussi à la croissance continue des taux d'activité au cours des trois dernières décennies ${ }^{(27)}$ et, plus particulièrement, aux taux d'activité féminine qui, pour les 25-54 ans, passent de $50 \%$ en 1970 à plus de $77 \%$ à partir de la moitié des années quatre-vingt-dix (Insee, Enquêtes emploi). Moins de femmes se retirent du marché du travail au moment du mariage ou à l'arrivée des enfants, et moins longtemps le cas échéant. Toutefois, l'activité féminine répond essentiellement au besoin d'une main d'œuvre « ajustable » de la part d'entreprises devant satisfaire des exigences croissantes de compétitivité et de résultats à court terme. La rationalisation de la production s'accompagne d'une contraction de la main d'œuvre sur des postes mobilisant des compétences moins spécifiques et l'économie se tertiarise. Les entreprises externalisent les tâches qui dépassent leur « cœur de métier » pour se dégager de certaines rigidités; la sous-traitance, les activités de service aux entreprises, se développent ; la main d'œuvre doit être plus polyvalente et plus flexible. 
À partir de la fin des années soixante-dix, le développement des «nouvelles formes d'emploi» notamment du temps partiel, a ainsi contribué à ce que les femmes participent au marché du travail, mais les a, par là même, orientées vers des emplois précaires. Aussi, les femmes salariées sont-elles encore six fois plus nombreuses à travailler à temps partiel que les hommes ${ }^{(28)}$ et, de façon générale, plus nombreuses en situation de précarité professionnelle, en inactivité subie ou au chômage ${ }^{(29)}$, leur taux de chômage étant supérieur de près de 2 points à celui de leurs « homologues » masculins (10,8\% contre $9,0 \%$ en 2005$)$.

$\mathrm{Au}$ fur et à mesure où les femmes ont intégré le marché du travail, elles se sont donc vues pour partie substituer des interruptions subies aux interruptions de carrière choisies - tout au moins consenties.

Si d'une génération à l'autre, les femmes sont donc plus nombreuses à suivre des carrières continues, elles restent minoritaires sur le «noyau dur » du marché du travail aux salaires élevés (groupe 7) ; elles en représentent moins du tiers des effectifs et le prolongement de leur activité les conduit plutôt à suivre des carrières aux salaires plus faibles (groupe 6) ou les amène sur des segments où elles sont plus vulnérables aux aléas conjoncturels (groupe 5).

\section{Conclusion}

L'analyse de classification des carrières des ayants droit du Régime général a mis en évidence une structure du secteur privé qui illustre les mutations intervenues sur le marché du travail au cours des dernières décennies. Le ralentissement économique, la réorientation des politiques de gestion de la main d'œuvre et les profondes transformations sociologiques, se traduisent notamment par un mouvement de précarisation, mais qui reste ciblé sur certaines catégories de salariés. Certains salariés semblent épargnés alors que d'autres cumulent les désavantages : chômage fréquent et de longue durée, et faibles rémunérations. Certes la grille d'analyse reste lâche, car seuls sept profils de carrière sont identifiés. Il en ressort néanmoins un dualisme qui oppose des salariés aux carrières continues et bien rémunérées occupant un emploi à temps complet en tant que cadre ou profession intermédiaire, à des travailleurs aux salaires plus faibles, éventuellement employés à temps partiel ou de façon intermittente, et qui éprouvent des difficultés à trouver un emploi régulier et connaissent plus fréquemment et plus durablement des périodes de chômage et d'inactivité. Ces derniers représentent les deux tiers des effectifs et supportent l'essentiel des transformations du secteur privé de ces dernières décennies.
La montée des tensions sur le marché du travail s'est traduite par un poids croissant des carrières les plus discontinues aux progressions salariales les plus lentes. Dans un contexte de rigidité à la baisse des salaires, l'ajustement s'est avant tout effectué par un recours accru aux emplois de courte durée, temporaires et à temps partiel, justement qualifiés de «flexibles » (L'Horty, 1999). Avec l'augmentation de l'activité féminine, la durée moyenne passée sur le marché du travail s'est allongée, mais au prix d'interruptions de carrière subies, plus courtes et plus fréquentes.

La bipolarisation entre, d'une part, des carrières précaires au profil salarial peu croissant et, d'autre part, des carrières continues aux progressions salariales plus soutenues, se maintient au fil des générations. Le clivage hommes-femmes la recoupe de moins en moins, mais l'évolution reste donc lente ${ }^{(30)}$. Certes, les modèles d'activité des hommes et des femmes convergent, mais non vers le standard de la carrière continue, mais vers un parcours plus discontinu ; celui des femmes s'améliore, alors que dans le même temps celui des hommes se dégrade (De Vroom, 2003).

Pour les générations de l'après baby boom, si le modèle de la carrière longue et continue du salarié du secteur privé persiste donc, il ne s'oppose désormais plus à celui d'une carrière brève où le retrait du marché du travail s'effectue précocement, mais à des parcours parfois plus longs et toujours plus discontinus. Aussi, comme l'ont noté Belloc et Lagarenne (1996), le développement des emplois « atypiques » constitue-t-il la principale remise en cause du modèle de la carrière continue. 


\section{Notes}

(1) Le Gall J.-M. (1992), La gestion des ressources humaines, Presses Universitaires de France, coll. «Que-sais-je ?», p. 23.

(2) En 2006, les régimes d'assurance vieillesse des artisans et des commerçants ont été réunis au sein du Régime Social des Indépendants (RSI).

(3) On renvoie entre autres aux travaux menés par Glaude (1986, 1987) sur l'enquête Emploi, Lhéritier (1992) et Bayet (1996a, b) à partir de l'enquête Structure des salaires ; Goux (1991) sur l'enquête Carrières et mobilité ; Bayet et Colin (1998), Guillotin et Hamouche (1998) et Koubi (2003) sur les DADS ; voir aussi sur cette dernière source, la revue de littérature de Francis Kramarz (2003).

(4) En 1997, $30 \%$ des salariés du secteur Industrie-Commerce-Services étaient employés dans des entreprises de moins de 20 salariés (Moussallam, 1999).

(5) Bien qu'annuellement, au plus quatre trimestres soient retenus pour le calcul de la pension (quelle qu'en soit la nature), l'ensemble des trimestres validés est néanmoins conservé dans le fichier (par exemple, 7 trimestres pour une année complète en chômage indemnisé).

(6) Des trimestres sont également accordés en cas de détention provisoire, pour le versement des indemnités de soins aux tuberculeux, ou encore pour la perception de l'allocation de préparation à la retraite pour les anciens combattants d'Afrique du Nord et d'Indochine.

(7) L'EIR est établi tous les quatre ans par la Drees qui interroge alors la quasi-totalité des caisses de retraite obligatoire, de base et complémentaire (une trentaine de régimes), sur la nature et le montant des prestations versées à un échantillon anonyme d'individus.

(8) En pratique, sont sélectionnés comme salaires correspondant à une activité régulière, les montants soumis à cotisations une année, pour laquelle aucune période non cotisée au Régime général (maladie, chômage, etc.) n'a été validée et la variation des salaires observés sur trois années successives ne laisse pas supposer la prise ou l'interruption de l'activité en cours d'année. Est ainsi exclu le salaire de l'année $t$ si le salaire reporté en $t+1$ (respectivement $t-1$ ) est supérieur (resp. inférieur) de plus de $25 \%$ à celui perçu en t et que l'année t-1 (resp. $t+1)$ ne fait l'objet d'aucun report de salaire (situation de prise d'activité en cours d'année). Pour les assurés de la génération 1935, ces critères conduisent à exclure en moyenne $8 \%$ des reports de salaire une année donnée, avec un maximum de $21 \%$ en 1956 (21 ans) lors de la période de service militaire.

(9) Sont exclus de l'analyse les comptes d'assurés qui n'ont acquis un droit à retraite que par le biais de l'Assurance vieillesse des parents au foyer (AVPF), sans cotiser, soit $2 \%$ des ayants droit pour la génération 1940.

(10) Les méthodes non hiérarchiques fournissent une classification à un seul niveau de partition et sont particulièrement adaptées au traitement de grands ensembles de données. Les méthodes hiérarchiques constituent des classes emboîtées ; par exemple, les algorithmes ascendants fonctionnent par regroupement successif de classes à chaque niveau.
Le principal inconvénient des procédures itératives non hiérarchiques est leur tendance à créer des groupes de taille égale, parfois au détriment de l'homogénéité des groupes. Une classification hiérarchique ascendante $(\mathrm{CAH})$ permet alors d'améliorer la partition initiale et offre, de surcroît, un certain confort dans le choix du nombre final de groupes, les classes produites étant imbriquées.

(11) Nombre imposé par les capacités informatiques alors disponibles.

(12) Les variables dépendantes sont donc les groupes de la typologie considérée, les variables explicatives, les séquences décrivant les carrières sur la période d'observation définie.

(13) En se limitant à la littérature francophone, on renvoie notamment aux travaux des sociologues Guillemard et Gaullier, et de l'économiste Gautié.

(14) La procédure statistique distingue en fait huit groupes, mais le $8^{\mathrm{e}}$ groupe a été fusionné au $7^{\mathrm{e}}$ en raison de la proximité de ses parcours professionnels et de son faible effectif (moins de $1 \%$ de la population).

(15) Cf. Briard (2006).

(16) En retenant des durées de carrières plus longues, les taux de mauvais classement sont les suivants : $22 \%$ pour la période $15-44$ ans, $15 \%$ pour la période $15-49$ ans, $11 \%$ pour la période $15-54$ ans, $10 \%$ pour la période $15-59$ ans.

(17) Quelques éléments sur l'évolution de la validation des périodes de maladie et d'invalidité sont présentés en annexe 3 .

(18) L'emploi « intermittent » est un emploi permanent sous contrat à durée indéterminé, mais qui comporte par sa nature (saisonnière notamment) une alternance de périodes travaillées et non travaillées.

(19) La dualité du secteur privé, rassemblant des salariés qui occupent des emplois stables à temps complet d'une part et des salariés aux parcours plus fragmentés d'autre part, n'est pas remise en question par ces lacunes et tend au contraire à être accentuée, la proportion des emplois aux conditions de travail atypiques étant probablement sous-estimée.

(20) L'estimation tient compte des changements de situation intervenus dans la carrière (indicatrice de mobilité). Elle est effectuée en contrôlant le sexe, le lieu de résidence, le secteur d'activité, la condition d'emploi et la catégorie socioprofessionnelle.

(21) Cf. note 5 .

(22) Cf. note 3.

(23) Selon l'Insee, en 2001, l'emploi salarié se répartissait entre les quatre grands secteurs d'activité comme suit : $2 \%$ dans l'Agriculture, $19 \%$ dans l'Industrie, $6 \%$ dans la Construction et $74 \%$ dans le Tertiaire (Insee, 2004 : T203B).

(24) L'exploitation de l'enquête Emploi Insee permet d'estimer qu'en 2001, parmi les salariés âgés d'au moins 60 ans relevant du Régime général, $19 \%$ étaient cadres, $41 \%$ employés et $79 \%$ travaillaient dans le Tertiaire.

(25) À partir de l'enquête sur le coût de la main d'œuvre et la structure des salaires de 1992, Araï et alii estiment les écarts de rémunération entre le travailleur moyen du secteur privé et le travailleur moyen d'un secteur d'activité donné selon une nomenclature à 15,40 ou 100 secteurs. Ils trouvent, par exemple, que la rémunération horaire du salarié du secteur de l'énergie est 1,3 fois supérieure à celle du salarié moyen si on ne tient pas compte des caractéristiques individuelles (expérience, diplôme, âge, sexe, régime horaire de travail, PCS, type de contrat de travail).

Les auteurs utilisent la nomenclature d'activités et de produits (Nap), laquelle est restée en vigueur jusqu'en 1992, avant d'être remplacée par la nomenclature des activités françaises (Naf) qui sert de support à notre étude. 
(26) De 1974 à 1977, le gouvernement français suspend l'immigration de travailleurs extracommunautaires, avant d'inciter financièrement puis d'organiser le retour de la main d'œuvre installée en proposant une aide au retour et en ne renouvelant pas les titres de séjour.

Dans sa brochure Regards sur l'immigration depuis 1945 (coll. «Insee Synthèses », n 30) parue en 1999, l'Insee indique (p. 64) que parmi les immigrés arrivés avant 1962 (les individus nés entre 1935 et 1940 avaient alors de 22 à 27 ans), 17,6 \% sont sortis de France entre 1968 et 1975 (soit au moins 6 ans plus tard), 17,2\% entre 1975 et 1982 . Parmi les immigrés arrivés entre 1962 et 1968 (ceux qui avaient 20 ans étaient alors nés entre 1942 et 1948), 29,0 \% sont sortis de France entre 1968 et 1975 et $18,1 \%$ entre 1975 et 1982 .

(27) En 1970, le taux d'emploi en France pour les 25-54 ans était de 73,8 \% ; il s'est stabilisé au-dessus de $86 \%$ à partir de la moitié des années quatre-vingt-dix (Insee, Enquêtes emploi).

(28) En 2002, 30,2 \% des femmes et 5,1\% des hommes travaillaient à temps partiel (Insee, Enquête emploi).

(29) En 2000, $21 \%$ des femmes actives - contre $15 \%$ des hommes - connaissaient dans l'année un changement de situation entre l'emploi, le chômage et l'inactivité (Insee, Enquête emploi).

(30) Pour une analyse plus complète sur cet aspect, on renvoie à nos travaux de recherche (Briard, 2006).

(31) La répartition n'est pas uniforme, car les classes médiane et maximale sont éclatées pour faire apparaître les valeurs caractéristiques que sont le $5^{\mathrm{e}}$ décile et le plafond. En outre, lorsque plus de $20 \%$ des assurés cotisent au plafond, la classe comprise entre le $8^{\mathrm{e}}$ décile et le plafond est vide.

(32) L'intention présidant à la définition de cet ordre est qu'en cas de coexistence de plusieurs états au sein d'une année civile, le codage conserve l'état le plus rare ou celui qui pourrait être masqué en raison de sa durée plus courte. Le passage par un autre secteur d'activité, la période de service militaire - laquelle s'étale au plus sur trois années, dont deux partiellement - et les périodes de maladie, sont ainsi codées prioritairement au chômage et le chômage de longue durée, prioritairement au chômage de courte durée. L'état « invalidité » est en revanche privilégié sur l'état « maladie», car jugé plus caractéristique que la perception d'indemnités journalières, laquelle pourrait d'ailleurs correspondre à une suspension du versement de la pension d'invalidité pour de simples raisons administratives.

(33) Le chômage de courte (resp. longue) durée prend ici une définition particulière : il désigne une période de chômage qui permet de valider moins (resp. plus) de trimestres que la moyenne de la génération au même âge.

(34) On pourra aussi trouver des techniques de « lissage » consistant, par exemple, à assimiler les séquences $\{\mathrm{xxxxyx}\}$ à $\{$ Xxxxxx\} (cf. Bigard et Guillotin, 1996).

\section{Bibliographie}

Amossé T. (2003). « Interne ou externe, deux visages de la mobilité professionnelle », Insee Première, n 921, 4 p.

Araï M., Ballot G. et Skalli A. (1996). « Différentiels intersectoriels de salaire et caractéristiques des employeurs en France », Économie et Statistique, n 299 , pp. 37-71.

Baudelot C. et Gollac M. (1997). « Le salaire du trentenaire : question d'âge ou de génération? », Économie et Statistique, $\mathrm{n}^{\circ}$ 304-305, pp. 17-35.

Bayet A. (1996a). "Carrières continues, carrières incomplètes et salaires ", Économie et Statistique, n 299, pp. 21-36.

Bayet A. (1996b). "L'éventail des salaires et ses déterminants », Données sociales Insee, pp. 190-198.

Bayet A. et Colin C. (1998). «Les évolutions individuelles de salaire sur dix ans sont difficiles à prévoir », Économie et Statistique, $\mathrm{n}^{\circ} 314$, pp. 3-24.

Becker G. S. (1975). Human Capital: a Theoretical and Empirical Analysis, $2^{\mathrm{e}}$ édition, Columbia University Press, New York.

Belloc B. et Lagarenne Ch. (1996). « Emplois temporaires et emplois aidés », Données sociales Insee, pp. 124-130.

Benzécri J.-P. (1981). Pratique de l'analyse des données : linguistique et lexicologie, Dunod, $565 \mathrm{p}$.

Bigard A. et Guillotin Y. (1996). "Carrières professionnelles, carrières salariales : la notion d'itinéraire caractéristique ", Économie et Statistique, n 299, pp. 73-89.

Briard K. (2006). Du marché du travail à la retraite : des trajectoires types pour les assurés du régime général de la Sécurité sociale, Thèse de doctorat en Sciences Économiques, Université Paris-Dauphine, 345 p.

Bry X. et Antoine P. (2004). " Explorer l'explicatif : application à l'analyse biographique », Population, vol. 59, $\mathrm{n}^{\circ}$ 6, novembre-décembre, pp. 909-946.

Chardon O. (2002). « De moins en moins d'inactifs entre la fin des études et l'âge de la retraite », Insee Première, $n^{\circ} 872$, $4 \mathrm{p}$.

Checcaglini A. et Lemaire R. (2001). «Transitions à l'entrée du marché du travail : construction d'une typologie des trajectoires des jeunes sortant d'apprentissage en Bretagne », $8^{e s}$ Journées d'études Céreq - Lasmas-IDL « Construction et usage des catégories d'analyse », $7 \mathrm{p}$.

Cloarec N. (2000). «Les passages de l'emploi à la retraite », Premières synthèses Dares, $\mathrm{n}^{\circ}$ 05.1, 11 p.

Colin Ch., Iéhlé V. et Mahieu R. (2000). « Les trajectoires de fin de carrière des salariés du secteur privé », Dossiers Solidarité et Santé Drees, n 3, pp. 9-23.

Concialdi P. et Ponthieux S. (1999). « Les bas salaires en France depuis le début des années 80 et quelques éléments de comparaison avec les États-Unis », Données sociales Insee, pp. 162-168.

Courgeau D. (1997). « Nouvelles approches méthodologiques en Sciences sociales : une vue d'ensemble », Population, vol. 52, n 4, pp. 793-802.

Courgeau D. et Guérin-Pace F. (1998). « Le suivi des itinéraires professionnels des couples par les méthodes de la statistique textuelle : lecture des parcours professionnels des couples », $4^{e s}$ Journées Internationales d'Analyse des données textuelles, Université de Nice Sophia Antipolis, pp. 221-232. 
Debrand T. et Privat A.-G. (2002). " L'évolution des carrières salariales au cours des cinquante dernières années "), Retraite et Société, n 36, pp. 188-201.

De Vroom B. (2003). "Ageing and Work: Trends, Concepts and Policies", in De Vroom B., Changing Labour Markets, Welfare Policies and Citizenship, pp. 37-50.

Dickens W. et Lang. K. (1985). “A Test of Dual Labor Market Theory", American Economic Review, vol. 75, n ${ }^{\circ}$, pp. 792-805.

Doeringer P. B. et Piore M. J. (1971). Internal Labor Markets and Manpower Analysis, Heath Lexington Books, Lexington (Mass.), 214 p.

Fénelon J.-P., Grelet Y. et Houzel Y. (1997). « Modéliser l'insertion », Formation Emploi, n 60, pp. 37-47.

Forgeot G. et Gautié J. (1997). « Insertion professionnelle des jeunes et processus de déclassement ", Économie et Statistique, $\mathrm{n}^{\circ}$ 304-305, pp. 53-74.

Galtier B. (1998). « Les trajectoires d'emploi des salariés à temps partiel dans le secteur privé ", Document de travail, $\mathrm{n}^{\circ}$ 98-04, Cserc.

Gaullier X. (1994). "La décennie de la retraite ", Gérontologie et Société, n 70, pp. 95-118.

Gautié J. (2003). « Transitions et trajectoires sur le marché du travail », Quatre pages, ${ }^{\circ} 59, \mathrm{CEE}$.

Germe J.-F. (pr.), Monchatre S., Pottier Fr. (rapp.) (2003). Les mobilités professionnelles : de l'instabilité dans l'emploi à la gestion des trajectoires, Commissariat Général au Plan, $112 \mathrm{p}$.

Glaude M. (1986). «Ancienneté, expérience et théorie dualiste du marché du travail ", Économie Appliquée, n 4 , pp. 847-876.

Glaude M. (1987). « La structure des salaires en 1985 », Données Sociales, Insee, pp. 159-171.

Goux D. (1991). « Coup de frein sur les carrières », Économie et Statistique, n 249, pp. 75-87.

Grelet Y. (2002). « Des typologies de parcours, méthodes et usages », Document Génération 92, n 20, Céreq.

Guillotin Y. et Hamouche S. (1998). « Capital humain et mobilité hiérarchique des salaires : une approche par les modèles de comptage ", Revue Économique, vol. 49, n 3 , pp. 901-911.

Koubi M. (2002). « Éléments de caractérisation des carrières salariales des générations nées entre 1908 et $1980 »$, Document de travail, $\mathrm{n}^{\circ} \mathrm{F} 0205$, Insee.

Koubi M. (2004). « Les carrières salariales par cohorte de 1967 à 2000 », Économie et Statistique, n 369-370, pp. 149-150.

Kramarz F. (2003). " Mobilité et salaires : une longue tradition de recherche », Économie et Statistique, $\mathrm{n}^{\circ}$ 369-370, pp. 113-118.

Lainé F. (1999). " Logiques sectorielles et nomenclature d'activités », Économie et Statistique, n 323, pp. 95-113.

Lebart L. et Salem A. (1988). Analyse statistique des données textuelles. Questions ouvertes et lexicométrie, Dunod, 209 p.

Le Minez S. (2002). « Topographie des secteurs d'activité à partir des flux de mobilité intersectorielle des salariés », Économie et Statistique, n 354 , pp. 49-83.

Lemistre P. et Plassard J.-M. (1998). « Des carrières salariales différenciées selon l'emploi occupé ", in Allouche J., Sire B. (eds), Ressources humaines une gestion éclatée, Economica, pp. 353-369.

Lhéritier J.-L. (1992). "Les déterminants du salaire », Économie et Statistique, n 257, pp. 9-21.
L'Horty Y. (1999). « Emplois flexibles, salaires rigides : dix faits stylisés en quête d'interprétations ", Contribution au groupe " emplois flexibles, salaire rigides ", contrat ACIT, réunion du 16/12/1999, $17 \mathrm{p}$.

Lollivier S. et Payen J.-F. (1990). « L’hétérogénéité des carrières individuelles mesurée sur données de panel ", Économie et Prévision, $\mathrm{n}^{\circ}$ 92-93, pp. 87-95.

McVicar D. et Anyadike-Danes M. (2002). "Predicting Successful and Unsuccessful Transitions from School to Work by Using Sequence Methods", Journal of the Royal Statistical Society, Series A, vol. 165, n², pp. 317-334.

Moussallam K. (1999). « Le poids des grandes entreprises dans l'emploi », Insee Première, $\mathrm{n}^{\circ}$ 683, 4 p.

Nauze-Fichet E. et Tomasini M. (2002). " Diplôme et insertion sur le marché du travail : approche socioprofessionnelle et salariale du déclassement », Économie et Statistique, $\mathrm{n}^{\circ}$ 354, pp. 21-48.

Osberg L., Apostle R. et Clairmont D. (1987). "Segmented Labour Markets and the Estimation of Wage Functions", Applied Economics, ${ }^{\circ}$ 19, pp. 1603-1624.

Pascal G. (2005). « Les retraités du régime général résidant à l'étranger », Retraite et Société, n 46, octobre, pp. 204-235.

Ponthieux S. (1997). « Débuter la vie active au milieu des années quatre-vingt-dix : des conditions qui se dégradent », Économie et Statistique, $\mathrm{n}^{\circ} 304-305$, pp. 37-51.

Vincens J. (1997). «L'insertion professionnelle des jeunes : à la recherche d'une définition conventionnelle », Formation Emploi, n $^{\circ}$ 60, pp. 21-43. 


\section{Annexe 1 : les techniques d'analyse typologique de parcours : codage et classification}

Les techniques d'analyse typologique des parcours consistent à rechercher des figures communes et récurrentes dans un ensemble de parcours observés empiriquement, chacun étant décrit par une ou plusieurs séquences d'états. Elles comprennent deux étapes : une phase de codage, puis une analyse de classification.

\section{Le codage des parcours}

La plupart des techniques d'analyse typologique de parcours représente le parcours comme une succession d'états codés par des caractères. Cependant, deux grands types d'approches se distinguent.

L'analyse de séquences (sequence analysis) est vraisemblablement la plus répandue. La méthode se décline sous de multiples formes autour de l'algorithme d'alignement optimal (OMA : Optimal Matching Algorithm). Cette méthode, développée en biologie moléculaire pour l'étude de la molécule d'ADN dans les années soixante-dix, fut propagée en sciences sociales par le sociologue A. Abbot. L'idée consiste à calculer le coût de transformer une séquence en une autre par des opérations de suppression, insertion ou substitution d'états. La matrice de coûts obtenue sur les différentes séquences observées est alors soumise à classification.

Les travaux des chercheurs français, notamment au sein du Céreq, Centre d'études et de recherches sur les qualifications, (cf. entre autres Checcaglini et Lemaire, 2001 ; Grelet, 2002), ou encore ceux de Guérin-Pace (1997), Courgeau et Guérin-Pace (1998), Bigard et Guillotin (1996), font plus largement appel à l'ensemble des techniques élaborées en analyse de données textuelles et diffusées par les ouvrages de Benzécri (1981) puis de Lebart et Salem (1988).

\section{La classification}

La classification a pour objet la recherche d'une partition ou d'une répartition des individus en classes ou catégories homogènes et les plus distinctes entre elles.

La méthode de regroupement adoptée dépend étroitement de la description des parcours réalisée au préalable et donc de l'objectif poursuivi. En effet, juger si des éléments sont proches «met en jeu à la fois les attributs sur lesquels on juge de la ressemblance et la façon dont on la mesure. » (Grelet, op. cit). Si les attributs sont quantitatifs, la mesure prend la forme de distance, s'ils sont qualitatifs, la mesure est un indice de similarité.

L'algorithme d'optimal matching fournit une matrice de distances entre séquences qui se désigne naturellement à l'application de méthodes de classification.

La description des parcours sous forme textuelle et leur découpage en " formes graphiques » (terme désignant une suite d'événements simples tels $\{\mathrm{ABA}\}$ ou $\{\mathrm{ABC}\})$ se poursuivent généralement par une analyse factorielle des correspondances multiples (AFCM). Les traitements statistiques portent alors sur les profils de fréquences des formes graphiques. L'analyse factorielle aide en outre à obtenir une partition stable : d'une part, elle réduit le nombre de variables considérées dans la classification ; d'autre part, elle produit des variables orthogonales ce qui limite l'apparition de biais ; enfin, elle épure les variables intervenant dans la classification d'éventuelles fluctuations aléatoires, celles-ci étant renvoyées dans les facteurs contribuant le moins à la variance de l'ensemble observé.

\section{- Les limites de la classification}

La classification présente un certain nombre de limites (cf. entre autres McVicar et Anyadike-Danes, 2002) qui tiennent pour l'essentiel à la sensibilité des résultats aux choix méthodologiques : celui de la méthode de classification - hiérarchique ou non hiérarchique -, celui de la distance retenue pour évaluer les proximités entre éléments à regrouper, et celui du nombre de groupes finalement constitués.

- Les stratégies de classification se scindent en deux grandes familles : les classifications non hiérarchiques et les classifications hiérarchiques, les deux pouvant être combinées.

Les méthodes non hiérarchiques fournissent une classification à un seul niveau de partition et sont particulièrement adaptées au traitement de grands ensembles de données. Les algorithmes itératifs (centres mobiles, k-moyennes, nuées dynamiques) produisent généralement des partitions imparfaitement superposables et tendent à produire des groupes de taille égale, parfois au détriment de leur homogénéité.

Les méthodes hiérarchiques constituent des classes emboîtées ; par exemple, les algorithmes ascendants fonctionnent par regroupement successif de classes à chaque niveau. Une classification ascendante hiérarchique $(\mathrm{CAH})$ enchaînée à une classification non-hiérarchique permet ainsi d'améliorer la partition initiale et offre, de surcroît, un certain confort dans le choix du nombre final de groupes, les classes produites étant imbriquées.

- Les critères de regroupement des algorithmes de classifications hiérarchiques (saut minimum, saut maximum, saut moyen, distances entre barycentres, saut de Ward) diffèrent sur la façon d'agglomérer des groupes d'individus et les groupes produits sont alors plus ou moins allongés ou sphériques.

- La décision du nombre de groupes final tient à la fois à des considérations d'ordres statistique et opérationnel. Le recours à des critères quantitatifs renseignant sur la perte ou le gain en homogénéité induits par la fusion de deux groupes ( $R^{2}$ semi-partiel, Cubic Clustering Criterion ...) peuvent alors aider à la décision et limiter l'arbitraire.

Finalement, afin d'évaluer la robustesse de la partition, une solution pragmatique consiste à procéder à des tests de sensibilité : appliquer différentes méthodes de classification, faire varier le nombre de groupes pour s'assurer de sa stabilité et utiliser différents sous-ensembles de données pour garantir sa reproductibilité (Fénelon et alii, 1997). 


\section{Annexe 2 : le codage des comptes individuels des assurés du Régime général pour une analyse typologique}

L'objet du codage - la traduction des états par des « lettres »- est de fournir une représentation compacte de la carrière en en sélectionnant les aspects les plus pertinents ; le choix d'une nomenclature d'états va cependant être largement guidé par les données disponibles. Le séquençage du parcours - c'est-à-dire son découpage en «mots » - est lui beaucoup plus dépendant de la problématique traitée ; la succession des étapes, la durée passée dans chacune d'elles et/ou la date à laquelle elles surviennent, pourront ainsi être privilégiées.

La procédure de codage adoptée peut finalement se résumer en trois étapes :

- la définition d'une nomenclature d'états annuels,

- son utilisation pour constituer un ensemble de modalités permettant de décrire la carrière fractionnée en périodes de cinq ans,

- puis la simplification de ce « vocabulaire » par une agrégation raisonnée des états annuels.

\section{Une nomenclature de 14 états annuels}

La carrière désigne ici la période retranscrite au compte de l'assuré du Régime général entre 15 et 60 ans. Les situations sont décrites par une nomenclature d'états annuels exclusifs (tableau 6). Elles sont de deux ordres :

- Les salaires (soumis à cotisations) témoignent d'une activité salariée effectuée dans le secteur privé. De façon à atténuer les effets conjoncturels qui pourraient biaiser une analyse intergénérationnelle, un codage reposant sur la distribution des salaires par génération et par âge est adopté. Sept classes sont définies de façon que, pour une année donnée, les individus d'une même génération soient répartis quasi uniformément ; les bornes supérieures des classes sont les $2^{\mathrm{e}}, 4^{\mathrm{e}}, 5^{\mathrm{e}}, 6^{\mathrm{e}}$ et $8^{\mathrm{e}}$ déciles, la dernière classe regroupant les salaires supérieurs ou égaux au plafond ${ }^{(31)}$

- Les périodes non cotisées relevant du Régime général (chômage, maladie, invalidité) et les périodes validées par les autres régimes, témoignent de périodes d'interruption de carrière dans le secteur privé.

Les situations simultanées sont exclues, soit parce qu'elles sont rares (simultanéité des périodes de chômage et de maladie la même année, par exemple), soit parce qu'elles reflètent une réalité partielle (salaire annuel reporté une année avec chômage ou maladie). Le codage privilégie alors les périodes d'interruptions de carrière sur les salaires avec, par ordre de priorité ${ }^{32}$, les périodes effectuées hors du secteur privé, celles validées au titre du service militaire, de l'invalidité, de la maladie, du chômage de longue durée, du chômage de courte durée.

Une description des carrières par période quinquennale est adoptée. Pour chacune des neuf sous-périodes qui constituent alors le parcours, sont dénombrées les différentes situations annuelles rencontrées par l'assuré et seule la temporalité des épisodes au sein de chacune de ces sous-périodes est perdue. Le détail infra-quinquennal n'intervient donc pas directement dans l'analyse de classification, mais la description des parcours des groupes qui en ressortent, reste menée sur un déroulement annuel.

\section{Tableau 6 : nomenclature des états annuels ${ }^{(31)}$}

\begin{tabular}{|ll|}
\hline & \multicolumn{1}{c|}{ Codification des salaires } \\
\cline { 2 - 2 }$S b$ & salaire euros ] 0, $2^{\mathrm{e}}$ décile ] \\
$S c$ & salaire euros] $2^{\mathrm{e}}$ décile, $4^{\mathrm{e}}$ décile ] \\
$\mathrm{Sd}$ & salaire euros ] $4^{\mathrm{e}}$ décile, médiane ] \\
$\mathrm{Se}$ & salaire euros] médiane, $6^{\mathrm{e}}$ décile ] \\
$S f$ & salaire euros ] $6^{\mathrm{e}}$ décile, $8^{\mathrm{e}}$ décile ] \\
$\mathrm{Sg}$ & Salaire euros] $8^{\mathrm{e}}$ décile, plafond [ \\
$\mathrm{Sh}$ & Salaire $\geq$ plafond \\
\hline & \multicolumn{1}{c|}{ Codification des périodes non cotisées } \\
\hline$U b$ & chômage de courte durée \\
$U h$ & chômage de longue durée* \\
$M$ & maladie, maternité, accident du travail (incapacité $<66 \%)$ \\
$I$ & invalidité \\
$G$ & service militaire, guerre \\
$A$ & trimestres validés par les régimes d'assurance vieillesse \\
$N$ & autres que le Régime général \\
\hline
\end{tabular}

(*) Le chômage de courte (resp. longue) durée prend ici une définition particulière: il désigne une période de chomage qui permet de valider moins (resp. plus) de trimestres que la moyenne de la génération au même âge.

\section{Fractionnement de la carrière en périodes quinquennales}

Après codage, les carrières se présentent sous la forme de séquences de 45 états (un pour chaque année de 15 à 59 ans). De façon à conserver la chronicité des différentes périodes de la carrière, tout en permettant un rapprochement des carrières erratiques, il est choisi de découper la carrière en neuf périodes de cinq ans. Cette amplitude semble raisonnable au regard de l'objectif d'une différenciation d'au plus une dizaine de groupes aux profils de carrière proches.

Un recodage est ensuite effectué ; il consiste à représenter la carrière par neuf séquences de quatorze caractères, le $j^{e}$ caractère désignant le nombre de fois où le $j^{e}$ état de la nomenclature a été rencontré au cours de la période concernée. Ainsi, pour une période donnée (15-19 ans par exemple), s'il apparaît deux fois l'état $S b$, et trois fois l'état $S c$, alors la période est décrite par la modalité $\{23000000000000\}$.

\section{Réduction du nombre de modalités : la simplification} du « vocabulaire »

La multiplicité des modalités issues du recodage rend nécessaire la réduction de la diversité des situations décrites afin de mener une analyse factorielle. La solution adoptée repose sur une réduction du nombre des états annuels utilisés pour former les modalités lorsque ces dernières sont peu représentées ${ }^{(34)}$.

L'élargissement du maillage de la nomenclature est effectué de façon itérative. Les modalités obtenues avec une nomenclature à 14 états et représentées à plus de $1 \%$ dans l'une des neuf périodes quinquennales sur l'ensemble de la population sont conservées. Les modalités restantes sont alors recodées en adoptant une nomenclature à 13 états, un 
nouvel état se substituant à deux des états de la nomenclature initiale. Seules les modalités représentées à plus de $1 \%$ sont alors conservées. La procédure est reconduite jusqu'à ce que toutes les modalités vérifient ce critère de représentativité minimale.

La procédure d'agrégation des états suppose d'établir un ordre de regroupement des états deux à deux.

$\mathrm{Si}$ on se restreint à la description des salaires, la nomenclature retient sept classes, établies par rapport à la distribution des salaires des assurés d'une même génération (cf. figure 11a). En notant $S a$, la classe correspondant à l'ensemble des situations de non-salariat dans le secteur privé (inactivité, maladie, etc.), on a, par construction, la relation : $S a<S b<\ldots<S h$.

La première agrégation d'états concerne les classes de salaires les plus élevés, la classe $S g$ étant vide lorsque le $8^{\mathrm{e}}$ décile et le plafond sont confondus. Par symétrie, il est ensuite procédé à l'agrégation des classes de salaires les plus basses $S b$ et $S c$, puis de classes à salaires élevés, $S e$ et $S f$, etc. (cf. figure 11b).

Dépourvue de structure d'ordre, la nomenclature décrivant les périodes validant de la durée d'assurance est simplifiée selon des considérations d'ordres divers :

- les états $A$ (autres régimes) et $N$ (inactivité) sont agrégés en premier lieu en raison des lacunes portant sur le report des trimestres validés par les autres régimes;

- le deuxième regroupement porte sur les deux états relatifs au chômage, le chômage de longue durée étant naturellement accolé à une ou deux périodes de chômage de plus courte durée ;
- le regroupement entre l'état $G$ (service militaire) et l'état $A N$, est également motivé par les lacunes de report des trimestres pour les périodes effectuées sous les drapeaux, mais aussi par le fait que ces périodes aient pu être validées par d'autres régimes que le Régime général ;

- les modalités $S, I M U$ et $A N G$, issues de la cinquième phase d'agrégation, permettent de distinguer l'activité salariée relevant du Régime général, des périodes d'interruptions de carrière prises en charge par le régime, des périodes hors du champ du régime ;

- enfin, à la sixième agrégation, ne subsiste plus que la distinction entre «salariat dans le privé» (état $S$ ) et « activité hors du secteur privé » (état $A N G I M U$ ).

Au terme de la procédure de recodages successifs, les carrières sont décrites par neuf modalités de deux à quatorze caractères selon les situations rencontrées par l'assuré. La modalité la plus courte permet de connaître le nombre d'années présentant le report d'un salaire au compte de l'assuré sur une période donnée (15-19 ans par exemple); la plus longue (14 caractères) renseigne sur l'ensemble des états décrits dans la nomenclature initiale.

En définitive, entre 15 et 59 ans, une description des carrières par période quinquennale est adoptée. Pour chacune des neuf sous-périodes qui constituent alors le parcours, sont dénombrées les différentes situations rencontrées par l'assuré et seule la temporalité des épisodes au sein de chacune de ces sous-périodes est perdue. Le détail infra-quinquennal n'intervient donc pas directement dans l'analyse de classification, mais la description des parcours des groupes qui en ressortent, reste menée sur un déroulement annuel.

Figure 11 : états pour la description des salaires

\section{a : nomenclature de référence}

$S a$ pas de salaire

$\mathrm{Sb}$ ] $0,2^{\mathrm{e}}$ décile]

Sc $] 2^{\mathrm{e}}$ décile, $4^{\mathrm{e}}$ décile]

$\mathrm{Sd}$ ] $4^{\mathrm{e}}$ décile, médiane]

$\mathrm{Se}]$ médiane, $6^{\mathrm{e}}$ décile]

Sf $] 6^{\mathrm{e}}$ décile, 8 ] décile]

$\mathrm{Sg}$ ] 8 décile, plafond[

$S h \geq$ plafond

\section{b : procédure d'agrégation}
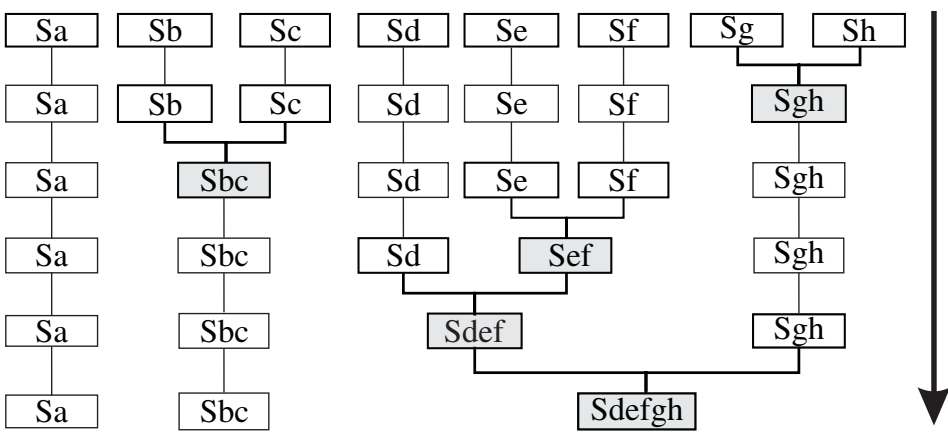

Figure 12 : états pour la description des périodes validant de la durée d'assurance

\section{$a:$ nomenclature de référence}

$S \quad$ salariat dans le secteur privé

Ub chômage de courte durée

Uh chômage de longue durée

$M$ maladie

$I \quad$ invalidité

$G$ service militaire, guerre

$A$ trimestres validés hors du sescteur privé

$N$ inactivité ou absence d'information

\section{b : procédure d'agrégation}
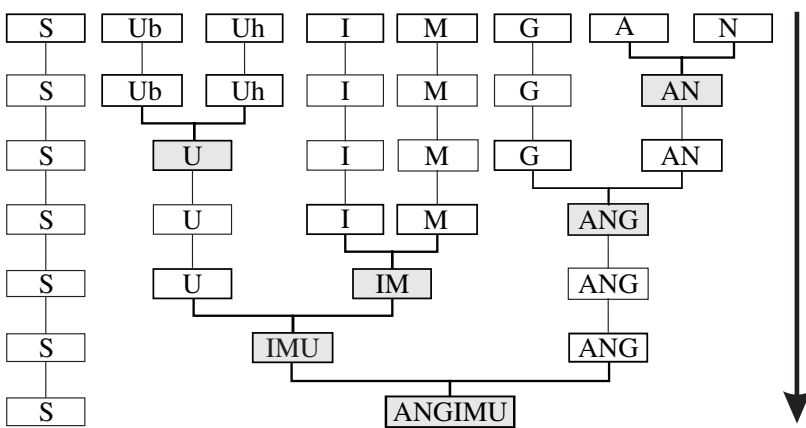
Les périodes de maladie, de maternité ou les accidents du travail entraînant une incapacité inférieure à $66 \%$, donnent lieu à la validation de trimestres de durée d'assurance par assimilation à des périodes de cotisation. Ces périodes, regroupées ici sous le vocable de «PA-maladie», correspondent à des interruptions de carrière qui interviennent généralement sans qu'il y ait rupture du contrat de travail. Lorsque les entreprises se substituent à la caisse d'assurance maladie pour le paiement des indemnités journalières (situation de subrogation), ces périodes n'apparaissent d'ailleurs pas sur le compte individuel de l'assuré du Régime général.

À un âge donné, les hommes salariés du secteur privé qui présentent le report d'une PA-maladie sont en proportion relativement stable d'une génération à l'autre (figure 13). À l'inverse, des modifications importantes peuvent être notées pour les femmes : la distribution des périodes assimilées au cours de la carrière met en évidence les périodes d'interruption pour cause de maternité et, par conséquent, les changements intervenus en matière de fécondité. Le recul de l'âge moyen de fécondité se manifeste par un étalement des naissances avec les générations 1946 à 1950, mais un âge modal qui reste sensiblement le même que celui des générations précédentes, soit 22 ans. Les générations suivantes marquent une rupture : l'âge modal passe à 26 ans pour les générations 1951 à 1955,27 ans pour les générations 1956 à 1960 ; recul de l'âge de maternité qui s'accompagne d'un étalement des naissances pour ces dernières.

La validation de trimestres pour invalidité ou pour des accidents du travail entraînant une incapacité supérieure à $66 \%$ est peu sensible aux effets de génération (figure 14). La part de la population concernée croît en revanche de façon continue avec l'âge et, au-delà de 55 ans, les hommes sont plus touchés que les femmes. La pénibilité de certains travaux effectués exclusivement par des hommes, dans le secteur du bâtiment ou de l'industrie notamment, explique vraisemblablement en grande partie cette différence.

Figure 13 : proportion de salariés du secteur privé validant des PA-maladie*

hommes

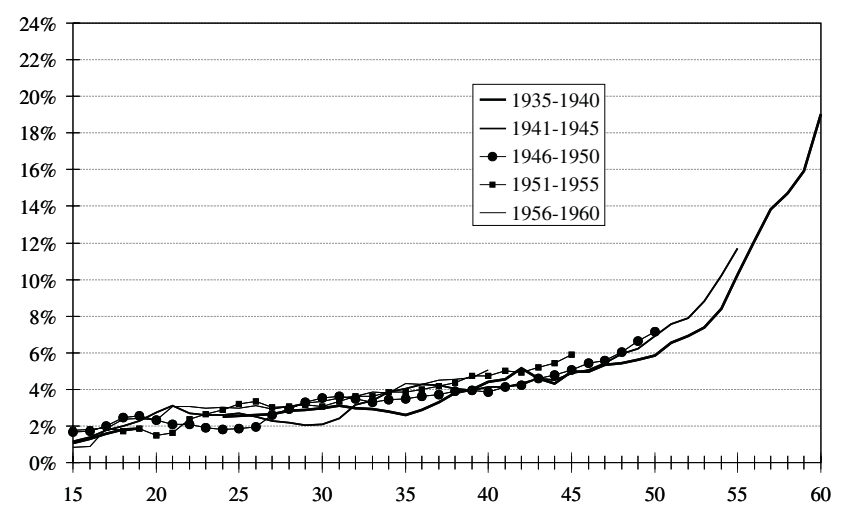

femmes

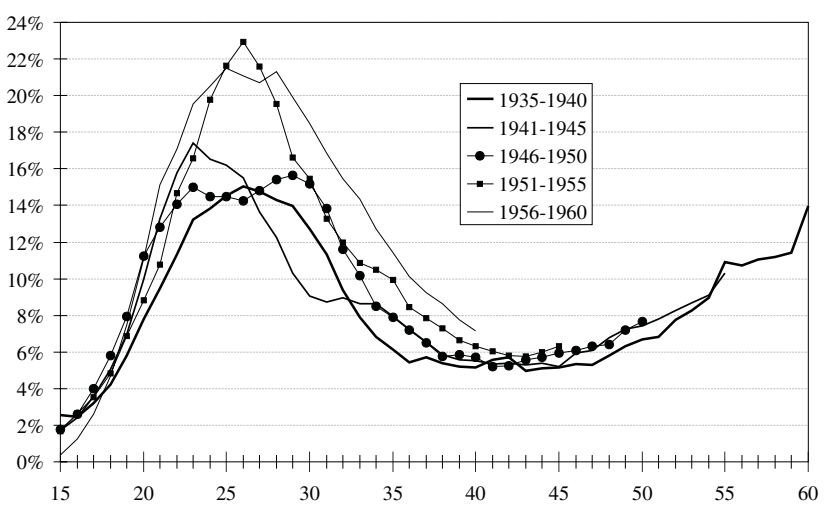

Champ : 500000 assurés du Régime général nés entre 1935 et 1960.

* Ratio entre le taux de report de PA-maladie et le taux de report de salaire correspondant à une activité régulière.

$P A$-maladie = trimestre d'assurance validé au titre de la maladie, de la maternité ou d'un accident du travail ayant entraîné une incapacité inférieure à $66 \%$.

Figure 14 : proportion d'assurés validant des PA-invalidité

hommes

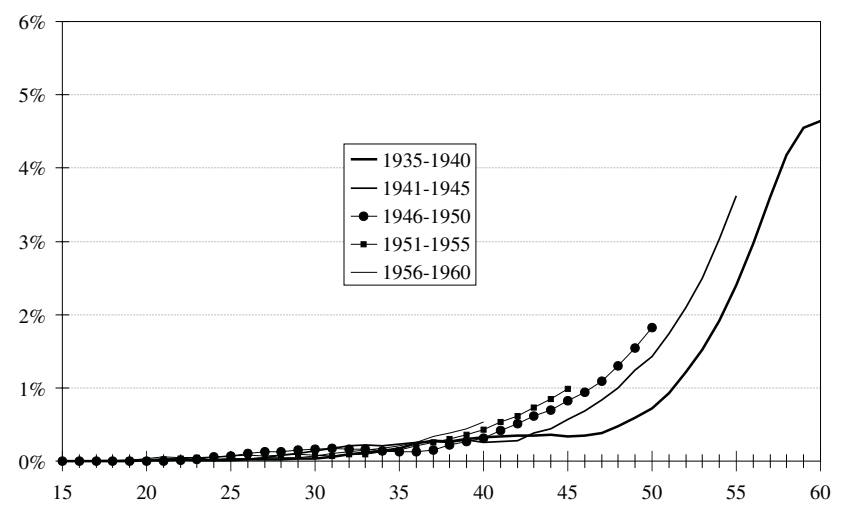

Champ : 500000 assurés du Régime général nés entre 1935 et 1960. femmes

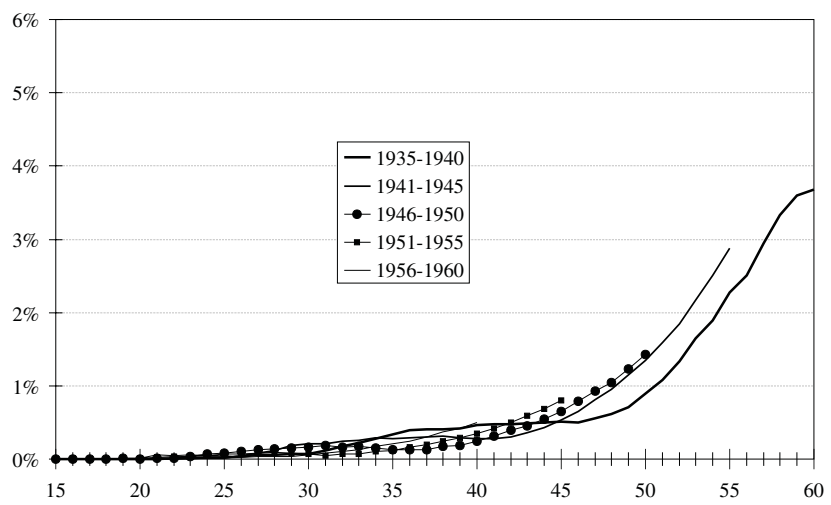


